\title{
Les Entreprises Implantées au Cameroun Manipulent-ils Leurs Résultats Pour Eviter de Publier les Petites Pertes?
}

\author{
Serge Christian SAHA ${ }^{1 *} \quad$ Louis NDJETCHEU ${ }^{2}$ \\ 1.Laboratoire d'Economie et de Management Appliquée, Université de Douala, Cameroun \\ 2. Faculté des Sciences Economiques et de Gestion Appliquée, Université de Douala, Cameroun
}

\begin{abstract}
The objective of this article is to determine if companies located in Cameroon manipulate their earnings to avoid publishing low losses. Based on a sample of 1,525 companies observed over a three-year period and using statistical tools such as normality histograms and statistical tests, we arrive at the results below. First, we note that there is indeed a discontinuity in the distribution of corporate earnings at the threshold of zero. This discontinuity allows us to identify suspicious companies which represent more than $67 \%$ of the companies studied. Also by comparing the number of suspicious businesses located to the right of the threshold to the number of suspicious businesses located to the left of the threshold, we find that there are more suspicious enterprises to the right of the zero earnings threshold. However, unlike studies carried out in European and American contexts, the proportion of suspicious companies located to the left of the threshold is relatively large. We then deepened our research to try to identify among the suspicious companies those which can be qualified as manipulative. This deepening was done through an analysis of the evolution over three years of the earnings of suspicious companies knowing that manipulation refers to the transfer over time of charges and products to obtain short terms changes in the transfer of wealth. This analysis allowed us to obtain, among the suspicious companies, a handling rate of more than $58 \%$. Keywords: earnings management, accounting thresholds, zero result, suspicious companies, manipulative companies.
\end{abstract}

DOI: $10.7176 /$ RJFA/11-2-06

Publication date: January $31^{\text {st }} 2020$

\section{Résumé}

L'objectif de cet article est de déterminer si les entreprises implantées au Cameroun manipulent leurs résultats pour éviter de publier les faibles pertes. Sur la base d'un échantillon de 1525 entreprises observées sur une période de trois ans et à l'aide des outils statistiques tels que les histogrammes de normalité et les tests statistiques, nous aboutissons aux résultats ci-après. Tout d'abord, nous constatons qu'il existe bien une discontinuité dans la distribution des résultats des entreprises au seuil du résultat nul. Cette discontinuité nous permet d'identifier les entreprises suspectes qui représentent plus de $67 \%$ des entreprises étudiées. Aussi en comparant le nombre d'entreprises suspectes situées à droite du seuil au nombre d'entreprises suspectes situées à gauche du seuil, nous constatons qu'il y a plus d'entreprises suspectes à droite du seuil du résultat nul. Toutefois à la différence des études effectuées dans les contextes européens et américains, la proportion des entreprises suspectes situées à gauche du seuil est relativement importante. Nous avons ensuite approfondis notre recherche pour tenter d'identifier parmi les entreprises suspectes celles qui peuvent être qualifiées de manipulatrices. Cet approfondissement a été fait à travers une analyse de l'évolution sur trois ans du résultat des entreprises suspectes sachant que la manipulation renvoie au transfert dans le temps des charges et des produits pour obtenir à court des modifications dans le transfert des richesses. Cette analyse nous permis d'obtenir parmi les entreprises suspectes, un taux de manipulation de plus de $58 \%$.

Mots clés : gestion des résultats, seuils comptables, résultat nul, entreprises suspectes, entreprises manipulatrices.

\section{Introduction}

Idéalement, les gestionnaires utilisent leur connaissance de la situation économique actuelle des entreprises pour donner une véritable image fidèle de la situation financière et de la performance de l'entreprise. Pour être utile à la prise de décision, l'information produite doit remplir à la fois les critères de pertinence et de fiabilité. Le but du référentiel comptable en vigueur qui guide et limite les gestionnaires dans leur production de l'information financière est précisément d'améliorer la pertinence et la fiabilité de l'information comptable et financière.

Malheureusement, le référentiel accorde aussi de la flexibilité dans l'application des normes comptables. C'est cette flexibilité qui est utilisée par les dirigeants pour éroder la fiabilité de l'information comptable. La nature discrétionnaire de cette pratique rend difficile sa détection. Une des approches de détection consiste à étudier la distribution statistique des résultats au voisinage des seuils.

L'approche de détection de la manipulation des chiffres comptables par les seuils examine les propriétés statistiques des résultats pour identifier un comportement qui influence les résultats. Ces études mettent l'accent sur le comportement des résultats autour d'un indice de référence déterminé, comme le résultat zéro ou d'un trimestre précédent, pour vérifier si l'incidence des montants au-dessus et en dessous de l'indice de référence est 
répartie de façon régulière ou reflète les discontinuités liées à l'exercice de la discrétion.

Une des stratégies de publication des résultats comptables consiste à gérer ces résultats afin d'atteindre certains seuils tel que l'objectif d'éviter un résultat négatif, d'éviter la baisse dans les séries de bénéfices et enfin de rejoindre les prévisions des analystes financiers. La manipulation des chiffres comptables afin d'atteindre certains seuils est liée au besoin psychologique des dirigeants d'atteindre les trois seuils précités. Pour satisfaire ces besoins, les résultats sont gérés pour atteindre certains bénéfices cibles appelés seuils. Ainsi, une banque accorde difficilement des prêts lorsque les résultats sont négatifs, les propriétaires de la firme n'accordent une prime à l'équipe dirigeante que si les résultats comptables dépassent un seuil fixé à l'avance et enfin, les analystes financiers ne recommandent une entreprise à l'achat que si ses bénéfices rejoignent ou s'approchent de leur estimations consensuelles.

Burgsthaler et Dichev (1997), Degeorges et al (1999) ont mis en évidence que les résultats comptables sont gérés afin de rejoindre les prévisions des analystes financiers. Ce comportement est compatible avec une pratique de manipulation des résultats comptables pour atteindre ou excéder le seuil psychologique que constitue le marché. Cela étant, l'objectif d'excéder les prévisions des analystes financiers peut être atteint de deux manières : soit par une manipulation des résultats à la hausse soit par l'influence des prévisions des analystes elles même à la baisse (Vidal, 2010). C'est ainsi que Burgstahler et Eames (1999) ont mis en évidence la manipulation des prévisions des analystes financiers par les dirigeants en prenant comme proxy liée à cette manipulation les changements dans les prévisions. Enfin, Hayn (1995), Burgstahler et Dichev (1997) ainsi que Degeorge et al (1999) ont testé empiriquement les deux seuils suivants: Le besoin d'éviter les pertes et le maintien d'une série de bénéfices en croissance continue. Les résultats des études précédentes attestent bien l'existence des seuils précités dans la série de bénéfices étudiés. Ainsi, les résultats situés juste au dessous des seuils seront manipulés à la hausse, alors que ceux très éloignés de ces seuils seront manipulés à la baisse. Ce qui rendra plus facile l'atteinte de ces seuils dans le futur.

La gestion des résultats par les seuils est certes une voie intéressante dans la littérature comptable dans aux Etats-Unis, en Europe, en Asie. Mais elle a comparativement fait l'objet de peu d'attention dans les pays africains. Au Cameroun, par exemple les rares études sur les seuils, à notre connaissance sont celles de Elle (2018) et de Ngantchou et Elle (2018). Or s'il est admis que dans le contexte camerounais, la principale motivation de la manipulation des comptes soit liée à la volonté de minimiser les transferts fiscaux, le contexte peut être très enrichissant pour tester la pertinence du seuil du résultat nul par exemple. L'objectif de la présente recherche est de prolonger l'intérêt de l'étude de la gestion des résultats par les seuils pour le contexte africain. En particulier les études ci-dessus ont la faiblesse de mobiliser l'approche par les seuils uniquement comme démarche méthodologique et non comme véritable sujet de recherche. Pourtant il peut être intéressant de questionner la dynamique des motivations des entreprises au Cameroun au voisinage du seuil Zéro. En effet si nous admettons que les entreprises dans le contexte du Cameroun manipulent leurs comptes pour minimiser les transferts fiscaux, on peut douter de l'intérêt d'une manipulation pour éviter de publier les faibles pertes.

Aussi, les études antérieures confondent les entreprises suspectes aux entreprises manipulatrices. Or toutes les entreprises se trouvant juste à droite ne sont pas forcement les entreprises manipulatrices. Il est possible que parmi ces entreprises, il y ait des entreprises n'ayant pas manipulés leurs comptes. C'est pourquoi, dans cette recherche, une originalité consiste à faire une démarcation nette entre les entreprises suspectes et les entreprises manipulatrices. Une autre originalité de notre étude consiste à étudier également le comportement des entreprises situées immédiatement à gauche du seuil du résultat nul pour détecter une manipulation éventuelle.

La suite de cet article est organisée de la manière suivante. Dans une première section, nous ferons une revue de la littérature, la deuxième section présentera nos choix méthodologiques, la troisième section est consacrée aux résultats et à la discussion. La recherche débouche sur une conclusion.

\section{Revue de la littérature}

L'approche de distribution, est relativement simple à utiliser, et c'est une description graphique des résultats après la manipulation des résultats déclarés. En outre, cette approche détecte la manipulation des comptes tout en évitant la question de l'erreur de mesure et de la spécification manquante résultant des modèles de manipulation des chiffres comptables selon les accruals (Sun et Rath, 2010). McNichols (2000) estime que les modèles de distribution sont puissants dans la détection de la manipulation des chiffres comptables, car ils fournissent aux chercheurs une forte prédiction sur le groupe d'entreprises comportant les manipulateurs à défaut d'estimer les accruals discrétionnaires. En outre, elle est considérée comme une méthode appropriée et puissante pour détecter la manipulation des chiffres comptables lorsqu'un nombre énorme d'entreprises sont susceptibles de manipuler les résultats.

Cependant, certaines études empiriques remettent en question les formes des distributions des résultats comme preuve d'absence / présence de la manipulation des chiffres comptables ne soutiennent pas l'hypothèse que la manipulation des chiffres comptables peut s'expliquer complètement par la discontinuité de la répartition des résultats. Dechow et al. (2003) ne trouvent pas d'association entre les accruals discrétionnaires et la discontinuité 
des résultats.

Aussi Durtschi et Easton (2009) fournissent des résultats qui devraient être pris en compte lors de l'utilisation des formes des fréquences de distributions des résultats comme méthode de détection de la manipulation des chiffres comptables. Ils montrent que ces formes peuvent être induites par la déflation, le choix de l'échantillon et une différence entre les caractéristiques des résultats obtenus par Beaver et al. (2007). Durtschi et Easton (2009) soulignent que les chercheurs devraient considérer que cela se reflète dans les formes de distribution et devraient nuancer l'interprétation de la discontinuité dans la distribution des résultats en tant méthode détection de la manipulation des chiffres comptables. Jacob et Jorgensen (2007) ne sont en mesure de remettre en cause les résultats de Burgstahler et Dichev (1997) en indiquant que ces résultats ne sont pas induits par la mise à l'échelle. Tout au plus, ils soutiennent que cette approche peut indiquer l'existence de la pratique de manipulation des chiffres comptables, mais ne permet pas de comprendre les techniques ou l'ampleur de la manipulation des chiffres comptables.

\subsection{De la suspicion à la confirmation de la manipulation}

La méthodologie des seuils facilite la mise en évidence de la propension de certaines entreprises rechercher l'atteinte d'un niveau précis de résultat. Nous opérerons une analyse de la distribution des résultats au voisinage des seuils comptables que nous identifierons. Notre analyse sera affinée à mesure des estimations qui renforceront nos hypothèses de travail pour permettre l'identification des entreprises manipulatrices. Ceci dit, une comparaison des résultats obtenus avec les résultats attendus sera également la bienvenue. Nous allons nous inspirer de la démarche des auteurs de référence dans la littérature : Burgstahler et Dichev (1997), Degeorge et al. (1999), (Mard, 2004) et Grima (2017). Selon ces auteurs il faut à analyser la distribution des résultats au voisinage du seuil de zéro afin de détecter les entreprises qui manipulent leurs résultats pour éviter les petites pertes, et les baisses de résultats.

\subsection{La démarche de détection des entreprises suspectes}

Le dessein ultime est de vérifier l'existence d'une anomalie dans la distribution du résultat au voisinage du seuil de zéro. Cette anomalie est identifiée par le truchement des histogrammes de normalité et de la courbe de la loi normale. Les entreprises, dont la distribution des résultats ne s'ajuste pas à la loi normale et qui sont à l'origine de la discontinuité dans la distribution des résultats sont considérées comme suspectes. Ces entreprises concentrées au voisinage du seuil sont suspectées de manipulation des nombres comptables pour éviter de publier les pertes. Pour ce faire, l'estimation de la distribution normale des résultats au seuil zéro est comparée à la distribution réelle. Les observations théoriques dans les intervalles de distribution sont calculées à travers la moyenne arithmétique des observations adjacentes à un intervalle donné (Burgstahler et Dichev, 1997). Le nombre d'observations attendu dans un intervalle précis est la somme des observations des intervalles adjacents, divisée par 2 comme l'indique la formule suivante :

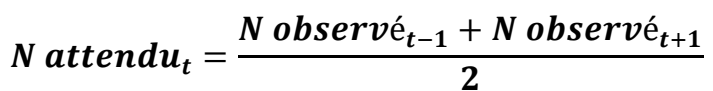

Où :

- $\quad \quad \quad$ attend $u_{t}$ est le nombre d'observations attendu pour l'intervalle $t$;

- $\quad N$ observé $t_{-1}$ est le nombre d'observations attendu pour l'intervalle t-1 ;

- $\quad N$ observé $t+1$ est le nombre d'observations attendu pour l'intervalle $t+1$.

L'écart entre l'effectif des observations attendues et l'effectif des observations réelles permet de valider les suspicions de manipulation.

\subsection{La démarche de détection des entreprises manipulatrices}

En considérant la manipulation comme un transfert des charges et des produits d'une période à l'autre, une analyse confirmatoire consiste à étudier la trajectoire du résultat avant impôt des entreprises suspectes situées à droite du seuil sur les périodes subséquentes. En clair, une baisse du résultat en $\mathrm{N}+1$ confirmerait que le résultat de $\mathrm{N}$ a été manipulé à la hausse, si en $\mathrm{N}+2$ par contre il y a une nouvelle hausse du résultat. Le décalage concernerait la période allant de $\mathrm{N}$ à $\mathrm{N}+1$. Nous avons emprunté cette démarche à Grima (2017). Aussi, il est bien admis dans la littérature que la manipulation des chiffres comptables s'opère à travers le transfert des charges et des produits dans le temps afin d'influencer à court terme la répartition de la richesse créée. Cette démarche peut être soustendue par cette affirmation du philosophe Raymond SMULLYAN : "To know the past, one must first know the future $»^{l}$

Ayant présenté les démarches d'identification des entreprises suspectes, il est urgent de révéler la variable comptable la plus représentative des chiffres comptables. C'est cette variable qui sera utilisée pour opérationnaliser la détection de la manipulation via les seuils.

\footnotetext{
${ }^{1}$ Cité par Lev (2003) : Nous traduisons ainsi cette phrase par « Pour connaître le passé, on doit d'abord connaître le futur »
} 


\section{Méthodologie de la recherche}

Le premier indicateur qui intéresse les utilisateurs des nombres comptables est le résultat net comptable. Le résultat net comptable est obtenu à travers un processus complexe dont les caractéristiques ne sont pas du tout aléatoires. Il est la conséquence des opérations ayant eu un impact positif ou négatif sur le patrimoine de l'entreprise au cours d'un exercice par le truchement des décisions prises par les dirigeants. L'étude des seuils focalise l'attention sur la distribution des résultats des entreprises.

Toutefois dans le processus de production de l'information comptable, plusieurs résultats sont calculés par le système d'information comptable. Pour les besoins liés au terrain empirique, nous allons nous limiter à la catégorisation du système comptable OHADA puisqu'il s'agit du référentiel comptable en vigueur au Cameroun. Sachant que au moment où nous réalisons cette thèse les états financiers issues de la reforme de 2017 et entrée en vigueur le $1^{\text {er }}$ Janvier 2018 ne sont pas encore disponibles au Cameroun, notre étude se réfère aux données issues du référentiel en vigueur avant le $1^{\mathrm{er}}$ Janvier 2018. Ce ne remet aucunement en cause la portée de notre étude car notre étude garde sa pertinence compte tenu du fait qu'elle ne porte pas sur le référentiel comptable mais sur l'utilisation de la discrétion dans les choix, les estimations et les régularisations comptables par les dirigeants. Or cette discrétion reste d'actualité dans le nouveau référentiel comptable OHADA issue de la réforme de 2017.

La cascade des résultats calculés selon le référentiel comptable OHADA se présente ainsi qu'il suit : le résultat d'exploitation, le résultat financier, le résultat des activités ordinaires, le résultat hors activités ordinaires et le résultat. Le résultat d'exploitation est obtenu en retranchant des produits liés à l'exploitation les charges d'exploitation. Le résultat financier provient de la soustraction des charges financières aux produits financiers. Le résultat des activités ordinaires est la somme du résultat d'exploitation et du résultat financier alors le résultat HAO provient des activités n'ayant pas un lien avec les activités ordinaires de l'entreprise. Le résultat net est obtenu en ajoutant au résultat des activités ordinaires, le résultat hors activités ordinaires et en retranchant l'impôt sur le résultat et la participation des travailleurs.

Notons qu'en dehors de cette nomenclature, l'administration fiscale camerounaise impose aux entreprises le calcul d'un autre résultat appelé « résultat fiscal ». Ce qui crée de facto un autre résultat : « le résultat avant impôt ».

Curieusement le compte de résultat OHADA ne prévoit pas une rubrique sur le résultat avant impôt. Celui-ci peut être obtenu en additionnant le résultat des activités ordinaires et le résultat hors activités ordinaires. Le tableau suivant décrit la démarche de calcul des différents résultats. Nous l'avons emprunté à l'acte uniforme OHADA relatif au droit comptable et à l'information financière de 2017 car celui-ci est mieux présenté que celui de 2000 .

Tableau 1 : Compte de résultat du système normal

\begin{tabular}{|c|c|c|c|c|c|}
\hline REF & LIBELLES & $+1-$ & $\mathbf{N}$ & $\mathbf{N}+\mathbf{1}$ & $\mathbf{N}+2$ \\
\hline TA & Ventes de marchandises & + & & & \\
\hline RA & Achat de marchandises & - & & & \\
\hline RB & Variation de stocks de marchandises & + & & & \\
\hline XA & MARGE COMMERCIALE (Somme TA à RB) & & & & \\
\hline TB & Ventes de produits fabriqués & + & & & \\
\hline $\mathrm{TC}$ & Travaux, services vendus & + & & & \\
\hline TD & Produits accessoires & + & & & \\
\hline $\mathrm{XB}$ & CHIFFRE D'AFFAIRE ( $\mathrm{A}+\mathrm{B}+\mathrm{C}+\mathrm{D})$ & & & & \\
\hline $\mathrm{TE}$ & Production stockée ( déstockage) & + & & & \\
\hline $\mathrm{TF}$ & Production immobilisée & + & & & \\
\hline TG & Subventions d'exploitation & + & & & \\
\hline $\mathrm{TH}$ & Autres produits & + & & & \\
\hline TI & Transferts de charges d'exploitation & + & & & \\
\hline $\mathrm{RC}$ & Achats de matières et fournitures liées & - & & & \\
\hline $\mathrm{RD}$ & Variation de stocks de matières premières et fournitures liées & & & & \\
\hline $\mathrm{RE}$ & Autres achats & - & & & \\
\hline $\mathrm{RF}$ & Variation de stock d'autres approvisionnements & & & & \\
\hline $\mathrm{RG}$ & Transports & - & & & \\
\hline RH & Services extérieurs & - & & & \\
\hline RI & Impôts et taxes & - & & & \\
\hline RJ & Autres charges & - & & & \\
\hline $\mathrm{XC}$ & VALEUR AJOUTEE ( XA+RA+RB)+(Somme TE à RJ) & & & & \\
\hline RK & Charges de personnel & - & & & \\
\hline $\mathrm{XD}$ & EXCEDENT BRUTE D'EXPLOITATION (XC+RK) & & & & \\
\hline $\mathrm{TJ}$ & Reprise d'amortissement, de provision et dépréciations & + & & & \\
\hline RL & Dotations aux amortissements, aux provisions et dépréciations & - & & & \\
\hline $\mathrm{XE}$ & RESULTAT D'EXPLOITATION (XD+TJ+RL) & & & & \\
\hline
\end{tabular}




\begin{tabular}{|c|l|c|c|c|c|}
\hline REF & \multicolumn{1}{|c|}{ LIBELLES } & $+/-$ & $\mathbf{N}$ & $\mathbf{N + 1}$ & $\mathbf{N + 2}$ \\
\hline TK & Revenus financier et assimilés & + & & & \\
\hline TL & Reprise de provisions et dépréciations financières & + & & & \\
\hline TM & Transferts de charges financières & + & & & \\
\hline RM & Frais financiers et charges assimilées & - & & & \\
\hline RN & Dotations provisions et aux dépréciations financières & - & & & \\
\hline XF & RESULTAT FINANCIER (Somme TK à RN) & & & & \\
\hline XG & RESULTAT DES ACTIVITES ORDINAIRES(XE + XF) & & & & \\
\hline TN & Produits cessions d'immobilisations & + & & & \\
\hline TO & Autres produits HAO & + & & & \\
\hline RO & Valeurs comptables des cessions d'immobilisations & - & & & \\
\hline RP & Autres charges HAO & - & & & \\
\hline XH & RESULTAT HAO ( Somme TN à RP) & & & & \\
\hline RQ & Participation des travailleurs & - & & & \\
\hline RS & Impôts sur le résultat & - & & & \\
\hline XI & RESULTAT NET ( XG+XH+RQ+RS) & & & & \\
\hline
\end{tabular}

\section{Source : OHADA (2017b)}

L'on constate bien que le compte de résultat OHADA n'a pas prévu la rubrique du résultat avant impôt pourtant la plupart des référentiels comptables dans les contextes à forte influence fiscale sur les exigences de divulgation des états comptables, une rubrique est prévue dans le compte de résultat pour le résultat avant impôt. Le résultat avant impôt s'obtient en ajoutant au résultat des activités ordinaires, le résultat hors activités ordinaires. Ce résultat va subir des traitements extracomptables via des déductions des produits non imposables et les réintégrations des charges non déductibles selon l'administration fiscale. Le tableau 22 de la déclaration statistique et fiscale du Cameroun joue le rôle de formulaire de détermination du résultat fiscal.

Ce tableau établit clairement la démarcation entre le résultat comptable (résultat avant impôt) et le résultat fiscal. Le résultat est obtenu au gré des ajustements fiscaux consécutifs aux divergences entre le droit comptable et la législation fiscale sur le caractère déductible de certaines charges et sur la prise en compte de certains produits. C'est ainsi que certains charges enregistrées en comptabilité ne sont non déductibles lors de la détermination du résultat fiscal. C'est le cas notamment de certaines dotations aux amortissements, les amortissements comptabilisés mais réputées différés en période déficitaire, certains dotations aux provisions, les amendes et les pénalités fiscales, les pourboires et certains dons.

$\mathrm{Au}$ regard, du caractère non déductible de certaines charges, il est concevable, la manipulation des chiffres comptables intervienne avant le traitement extracomptable débouchant sur la détermination du résultat fiscal. En effet, les dirigeants ne sont pas ignorants des divergences de déductibilité entre la conception fiscale et la conception comptable des charges. La manipulation concernerait alors les charges dont la déductibilité fiscale n'est pas contestée aussi bien partiellement qu'entièrement en prenant soin d'éviter la fraude pour prévenir le risque de redressement fiscal et de contestation de l'administration fiscale (Breton et Schatt, 2003). C'est la raison pour laquelle, nous avons opté pour le résultat avant impôt comme étant la variable comptable dont la représentation de la distribution statistique permettra de détecter les manipulateurs. Naturellement, pour éviter la forte dispersion de la distribution, cette variable est mise à l'échelle à travers le rapport entre le résultat avant impôt (RAI) et le total de l'actif (TA) : RAI / TA. 
Tableau 2 : Tableau de passage du résultat comptable avant impôt au résultat fiscal

\begin{tabular}{|c|c|c|c|}
\hline & & Ligne & MONTANTS \\
\hline SOLDE DU & BENEFICE NET COMPTABLE AVANT IMPÔT & 1 & \\
\hline $\begin{array}{l}\text { RESULTAT NET } \\
\text { AVANT IMPÔT SUR } \\
\text { LE RESULTAT }\end{array}$ & PERTE NETTE COMPTABLE AVANT IMPÔT & 2 & \\
\hline
\end{tabular}

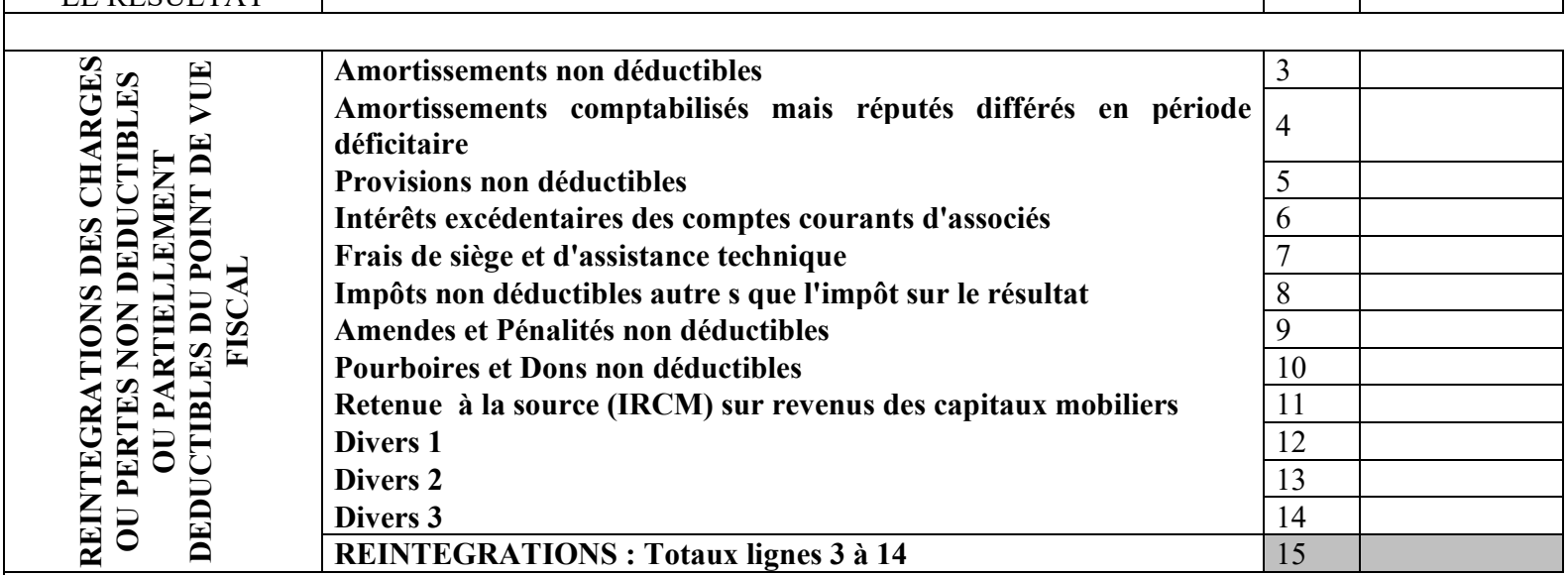

Total intermédiaire POSITIF : ligne $15+$ ligne 1 ou ligne 15 - ligne 2 Total intermédiaire NEGATIF : ligne 2 - ligne 15

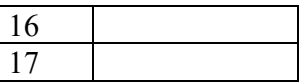

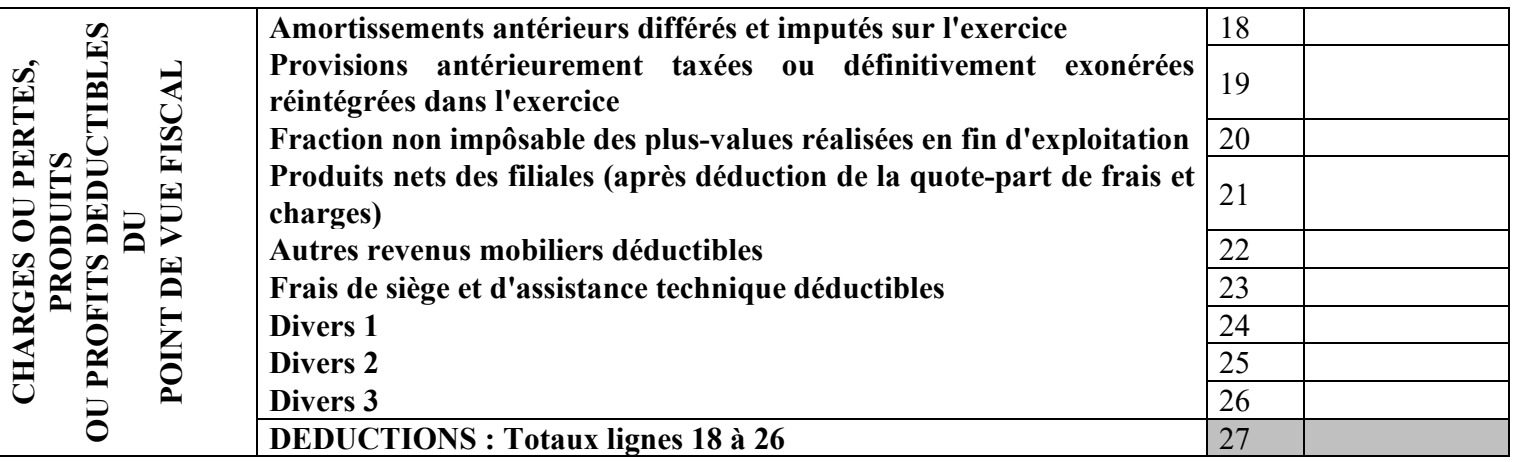

\begin{tabular}{|c|c|c|c|c|c|c|c|}
\hline \multirow{2}{*}{\multicolumn{2}{|c|}{ RESULTAT FISCAL }} & \multirow{2}{*}{\multicolumn{4}{|c|}{$\begin{array}{l}\text { BENEFICE FISCAL DE L'EXERCICE : ligne } 16 \text { - ligne } 27 \\
\text { PERTE FISCALE DE L'EXERCICE : ligne } 27 \text { - ligne } 16 \text { ou ligne } 17+ \\
\text { ligne } 27\end{array}$}} & \multirow{2}{*}{28} & \multirow[b]{3}{*}{\begin{tabular}{|l|}
$\begin{array}{l}\text { Principal de } \\
\text { l'Impôt }\end{array}$ \\
\end{tabular}} \\
\hline & & & & & & & \\
\hline \multirow{11}{*}{ 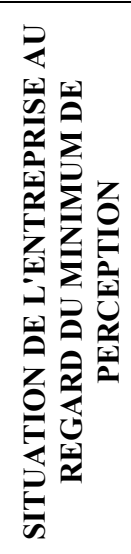 } & \multicolumn{3}{|c|}{ RUBRIQUES } & Ligne & Bases & Taux & \\
\hline & \multicolumn{2}{|c|}{ Minimum de Perception } & $\begin{array}{l}\text { Minimum } \\
\text { proportionnel au } \\
\text { chiffre d'affaires }\end{array}$ & 30 & & $2 \%$ & \\
\hline & \multicolumn{3}{|c|}{ Impôt sur les Sociétés } & 31 & & $30 \%$ & \\
\hline & \multirow{8}{*}{$\begin{array}{l}\text { Impôt } \\
\text { calculé } \\
\text { sur le } \\
\text { Bénéfice } \\
\text { fiscal de } \\
\text { l'Exercice } \\
\text { (ligne 30) }\end{array}$} & \multirow{8}{*}{$\begin{array}{l}\text { Impôt sur le revenu des } \\
\text { Entrepreneurs Individuels } \\
\text { Taxe proportionnelle }\end{array}$} & \multirow{7}{*}{$\begin{array}{l}\text { BIC et BNC } \\
\text { Bénéfices } \\
\text { Artisanaux } \\
\text { Bénéfices } \\
\text { Agricoles } \\
\text { Taxe } \\
\text { Proportionnelle }\end{array}$} & 32 & & & \\
\hline & & & & 33 & & & \\
\hline & & & & 34 & & $11 \%$ & \\
\hline & & & & 35 & & & \\
\hline & & & & 36 & & & \\
\hline & & & & 37 & & & \\
\hline & & & & 38 & & $15 \%$ & \\
\hline & & & $\begin{array}{l}\text { TOTAL lignes } \\
32 \text { à } 38\end{array}$ & 39 & & & \\
\hline
\end{tabular}

Source : DGI (2019)

Une fois les arguments ayant motivé le choix de la variable d'analyse mis en perspective, il est important de motiver le choix du seuil à utiliser parmi la liste des seuils validés par les études antérieures. Le choix du seuil dépend d'un ensemble de critères qu'il convient de mettre en lumière, le seuil étant la valeur à partir de laquelle se produit un phénomène ou en deçà de laquelle il ne se produit plus. 


\subsection{Le choix du seuil comptable approprié pour le contexte du Cameroun}

La littérature sur la détection de la manipulation des chiffres comptables a identifié trois principaux seuils : le seuil du résultat nul, la moyenne des prévisions des analystes financiers, le résultat de l'année précédente (Grima, 2017). Ces seuils ont surtout été développés dans les contextes européens et américains ; ce qui rends discutable leur applicabilité dans le contexte camerounais eu égard au fait que la dynamique des motivations à la manipulation n'est pas rigoureusement identique.

La moyenne des prévisions des analystes financiers traduit la volonté des entreprises à atteindre ou franchir les prévisions des analystes financiers afin de ne pas surprendre désagréablement les investisseurs qui se fient à ces prévisions dans l'allocation des financements. Or au Cameroun, l'évaluation des entreprises par les analystes financiers est une vue de l'esprit. Par conséquent, le seuil de la moyenne des prévisions des analystes financiers est inopérant pour le contexte du Cameroun.

Le seuil du résultat de l'exercice précédent renvoie à la volonté des entreprises de publier un résultat supérieur sinon égal à celui de l'exercice précédent afin de maintenir une tendance plus ou moins régulière dans l'évolution des résultats et de minimiser ainsi le risque lié aux actifs financiers émis par l'entreprise. Ce seuil semble recevable pour orienter la manipulation des chiffres comptables au Cameroun mais n'est pas représentatif car la dynamique des motivations de la manipulation épouse surtout la minimisation du transfert de la richesse des actionnaires vers l'Etat (Ngantchou et Elle, 2018).

Le seuil de résultat nul traduit la volonté des entreprises d'éviter de publier les faibles pertes en manipulant les comptes pour publier les faibles profits afin d'éviter la sanction des marchés financiers. Or au Cameroun, les états financiers sont principalement destinés à l'administration fiscale (Ngantchou, 2013). Ce qui réduit la portée du résultat nul comme seuil approprié. Toutefois, sachant que l'administration a neutralisé la portée d'un résultat négatif ou nul sur la valeur de l'impôt sur le revenu via l'algorithme du minimum de perception et que celle-ci dispose d'un pouvoir de contestation potentiellement nuisible pour les manipulateurs, la manipulation pour publier les faibles profits ou les faibles pertes peut représenter en enjeux moins risqué comparativement à la manipulation pour publier les grosses sachant que les pertes n'annulent pas les transferts fiscaux, le minimum de perception ayant intervenu en amont.

Au regard de ce qui précède, le résultat nul est le seuil le plus recevable pour notre contexte. D'ailleurs, les principales études ayant étudié la manipulation via les seuils au Cameroun ont invariablement adopté le seuil du résultat nul. Nous l'adoptons dans le cadre de cette thèse tout laissant le débat pendant pour les recherches ultérieures.

Ayant motivé nos choix relativement à la variable « résultat avant impôt » et au seuil du « résultat nul », qu'il nous soit permis de développer les hypothèses qui nous orienterons dans la suite de ce chapitre.

\subsection{Le développement des hypothèses testables}

Notre étude cible les distributions des résultats à l'approche du seuil de zéro afin de déterminer si les entreprises manipulent leurs résultats pour éviter de publier les faibles pertes et les fortes baisses de résultats. Notre analyse sera approfondie par une identification des entreprises manipulatrices. Depuis les travaux séminaux de Hayn (1995), un courant de la littérature sur la manipulation des chiffres teste l'hypothèse de la manipulation par les seuils. Ultérieurement, les travaux de Hayn (1995) ont été prolongés grâce à Burgstahler et Dichev (1997), Degeorge et al. (1999). Selon ces auteurs, le seuil permet de mettre en évidence une discontinuité dans la distribution des résultats après manipulation. Cette discontinuité traduit la volonté des entreprises à manipuler leurs comptes pour atteindre ou franchir le seuil. Trois principaux seuils ont été mis relief par les études antérieures : le seuil du résultat nul, le seuil des variations nulles du résultat et le seuil des prévisions des analystes financiers ou des dirigeants.

Plusieurs travaux empiriques ultérieurs ont testé l'hypothèse selon laquelle l'approche par les seuils permet de détecter la manipulation des comptes. Par exemple, Mard (2004), Grima (2017) trouvent que le seuil du résultat nul, le seuil des variations nulles du résultat, le seuil des prévisions des analystes financiers permettent de détecter la manipulation des nombres comptables dans l'environnement français. Sellami et Ajaoud (2010) trouvent les mêmes résultats dans l'environnement tunisien. Ngantchou et Elle (2018) quant à eux valide l'hypothèse de la pertinence du seuil du résultat nul en contexte camerounais.

La littérature sur les seuils comptables soutient que les entreprises préfèrent la publication d'un résultat faiblement positif au détriment d'un résultat faiblement négatif (Burgstahler et Dichev, 1997; Degeorge et al., 1999 ; Mard, 2004 ; Vidal, 2008 ; Grima, 2017 ; Ngantchou et Elle, 2018)

C'est en droite ligne avec les travaux empiriques ci-haut que nous formulons nos hypothèses sur la manipulation des chiffres comptables pour atteindre ou franchir des seuils précis de la manière suivante :

- H 1: L'approche par le seuil du résultat nul permet de détecter, les entreprises non suspectes, les entreprises suspectes et les entreprises manipulatrices;

- H 2 : La proportion d'entreprises suspectes se trouvant à droite du seuil du résultat nul est plus importante que celle se trouvant à gauche du seuil du résultat nul ; 


\subsection{L’approche par le seuil du résultat nul permet de détecter, les entreprises non suspectes, les entreprises suspectes et les entreprises manipulatrices.}

Conformément au consensus de la littérature sur les seuils, il est admis que la distribution des résultats non manipulée devrait suivre une distribution de la loi normale ou tout au moins une distribution régulière. Or les travaux séminaux de Burgstahler et Dichev (1997) mettent en évidence une discontinuité aux alentours de certains seuils. Pour le cas des entreprises camerounaises pour lesquels il y a une forte présomption de manipulation eu égard aux motivations fiscales, nous pensons que la concentration des entreprises autour du résultat nul sera forte traduisant un comportement de manipulation à l'approche du résultat nul. Les entreprises suspectes sont celles pour lesquelles la distribution des résultats n'obéit pas à la loi normale. Les entreprises non suspectes sont celles dont la distribution des résultats est conforme à une allure plus ou moins gaussienne. Alors que les entreprises manipulatrices sont une partie des entreprises suspectes dont l'analyse de leurs résultats trois années consécutives montre qu'ils ont effectivement manipulé leurs résultats.

\subsection{La proportion d'entreprises suspectes se trouvant à droite du seuil du résultat nul est plus importante que celle se trouvant à gauche du seuil du résultat nul}

Certaines recherches postulent laissent présager que la manipulation pour atteindre le résultat positif est marginale (Vidal, 2010b). Dans ces travaux la mesure de l'ampleur de la manipulation des nombres comptables est effectuée en faisant un rapprochement entre l'effectif immédiatement à droite du seuil et l'effectif immédiatement à gauche du seuil. Ainsi la sous représentation à gauche et la sur représentation à droite sont assimilées au même phénomène : la manipulation des chiffres comptables. La concentration des entreprises aussi bien à gauche que à droite du seuil sont les deux faces du même phénomène mais avec une forte probabilité de divergence dans les motivations de ceux qui font tout pour ne pas s'éloigner du seuil à défaut de le franchir.

Figure 2 : La présomption de manipulation marginale

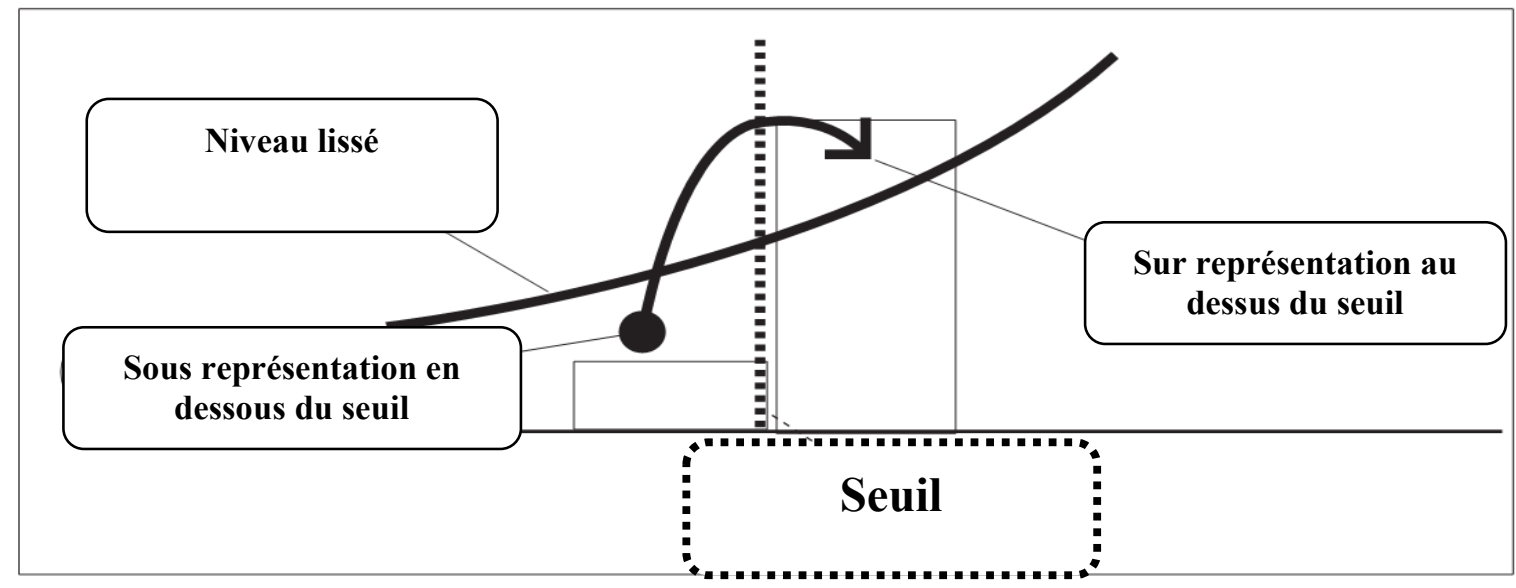

Source : Vidal (2010b)

Au-delà d'un simple évitement du seuil zéro, en fonction des avantages et des inconvénients, les entreprises peuvent manipuler leurs résultats pour publier des faibles profits. La littérature fait état d'une forte propension des entreprises à préférer la publication d'un résultat faiblement positif au détriment d'un résultat faiblement négatif (Burgstahler et Dichev, 1997 ; Degeorge et al., 1999 ; Mard, 2004 ; Vidal, 2008 ; Grima, 2017 ; Ngantchou et Elle, 2018). En effet une partie prépondérante des parties prenantes ne fait pas de différence entre les firmes publiant les résultats faiblement positifs et les firmes publiant les résultats négatifs. Pour cette raison, les entreprises ayant des résultats non manipulés faiblement négatifs préfèreraient les manipuler pour publier les faibles profits.

Dans une logique fiscale, afin de minimiser les transferts fiscaux, certaines entreprises peuvent, pour éviter de publier les forts profits, manipuler leurs résultats élevés pour publier des faibles profits. Ce qui leur permet de réduire la base imposable et donc de publier les faibles profits. Autrement dit, le transfert des effectifs peut aussi se faire des entreprises fortement bénéficiaires vers les entreprises faiblement bénéficiaires.

La plupart des recherches semble ignorer la possibilité que les entreprises puissent aussi manipuler leurs résultats pour éviter de publier les grosses pertes. En la forte représentation des entreprises faiblement bénéficiaires par rapport aux autres entreprises déficitaire traduirait un transfert irrégulier des entreprises vers la droite. Il est aussi possible que dans un souci de réduction des transferts fiscaux à une expression marginale ou nulle, que certaines entreprises ayant des résultats non manipulés positifs les manipulent pour publier les faibles.

Les recherches sur la manipulation des chiffres comptables sont réalisées à travers l'étude de la distribution des résultats au voisinage des seuils comptables. La méthodologie utilisée sera détaillé et les motivations de nos options méthodologiques expliqué à ce niveau. Ceci fait nos hypothèses sont présentés ainsi que les tests à effectuer. Pour une meilleure cohérence, nous présentons, d'abord, l'échantillon de notre étude et les sources de collecte des 
données.

\subsection{La constitution de l'échantillon et la collecte des données}

Notre base des données initiale est composée de 22181 observations (entreprises-années). Les détails concernant le nombre d'entreprises par années sont fournis dans le tableau subséquent. La population choisie représente les entreprises camerounaises sur la période allant de 2010 à 2015. Les données ont été recueillies à partir de la base de données l'institut national de la statistique du Cameroun (INS). Cette base regroupe des données comptables détaillées relatives à l'ensemble des entreprises au Cameroun. Notre échantillon comporte les entreprises de tous les secteurs d'activité selon la nomenclature classique : secteur primaire, secteur secondaire et secteur tertiaire. Ce qui nous permet de tenir compte de la structure de l'économie camerounaise. La répartition par secteur d'activité est par la suite présentée dans un tableau.

Notre processus d'échantillonnage se présente de la manière suivante :

- 1 ère étape : élimination des observations ayant des données manquantes compte tenu de leur indisponibilité; élimination des individus ayant des données aberrantes c'est-à-dire ayant un total actif (AT) nul ou négatif ;

- $\quad 2^{\mathrm{e}}$ étape : Recherche et exclusion éventuelle des entreprises à caractère financier (les banques, les institutions financières et les sociétés d'assurance) car leurs règles comptables sont spécifiques. Leur comptabilité n'est pas analogue à celle des sociétés industrielles et commerciales. Nous n'avons éliminé aucune observation à cause de ce critère car les données obtenues ne contiennent pas les entreprises à caractère financier.

- $\quad 3^{\text {e }}$ étape : Elimination des observations extrêmes selon le résultat avant impôt (RAI) et l'actif total (AT) : (RAI / AT supérieur ou égal à 1 et RAI / AT inférieur ou égal à -2 ).

Le tableau ci-dessous détaille la démarche utilisée pour constituer notre échantillon :

Tableau 3 : Processus d'échantillonnage des entreprises camerounaises

\begin{tabular}{|c|c|c|c|c|c|c|c|}
\hline & $\mathbf{2 0 1 0}$ & $\mathbf{2 0 1 1}$ & $\mathbf{2 0 1 2}$ & $\mathbf{2 0 1 3}$ & $\mathbf{2 0 1 4}$ & $\mathbf{2 ~ 0 1 5}$ & $\begin{array}{c}\text { Total des } \\
\text { observations }\end{array}$ \\
\hline Population disponible & $\mathbf{1 3 8 4}$ & $\mathbf{4 3 2 1}$ & $\mathbf{6 3 9 5}$ & $\mathbf{5 9 2 6}$ & $\mathbf{1 0 3 1}$ & $\mathbf{4 0 3 1}$ & $\mathbf{2 3} \mathbf{0 8 8}$ \\
\hline $\begin{array}{c}\text { Données manquantes et } \\
\text { Données aberrantes (Total } \\
\text { Actif = ou inf. à 0) }\end{array}$ & 13 & 52 & 111 & 74 & 14 & 207 & 471 \\
\hline $\begin{array}{c}\text { Données extrêmes RAI/AT } \\
\text { sup ou =1 }\end{array}$ & 10 & 31 & 52 & 48 & 5 & 32 & 178 \\
\hline $\begin{array}{c}\text { Données extrêmes RAI/AT } \\
\text { inf. ou =-2 }\end{array}$ & 15 & 45 & 62 & 96 & 8 & 32 & 258 \\
\hline $\begin{array}{c}\text { Banques, institutions } \\
\text { financières et sociétés } \\
\text { d'assurances }\end{array}$ & 0 & 0 & 0 & 0 & 0 & 0 & 0 \\
\hline Échantillon de départ & 1346 & 4193 & 6170 & 5708 & 1004 & 3760 & 22181 \\
\hline
\end{tabular}

Source : Par nos soins

Notre échantillon comporte un nombre d'entreprises qui varie sur la période d'étude. En effet ceci est la conséquence d'une conjonction de facteurs : la création de nouvelles entreprises, la disparition de certaines entreprises de la base de l'INS, la non transmission par certaines entreprises de leurs données comptables à l'INS, la non numérisation de certaines données par l'INS du fait des incohérences dans les données.

Les contraintes liées à l'homogénéité de l'échantillon sur toute la période d'étude nous imposent de créer une cohorte. Nous avons fait plusieurs simulations pour obtenir une cohorte. La première cohorte sur la période 2010 - 2015, nous a donné 42 entreprises. La deuxième cohorte sur la période 2010 - 2014 a donné 108 entreprises. La troisième cohorte sur la période 2010 - 2013 a donné 522 entreprises. Les différentes simulations nous ont finalement permis d'obtenir une cohorte maximale de 1525 entreprises si nous choisissons la période $2011-2013$. C'est ce qui justifie que pour la suite des analyses, nous sommes obligés de prendre la période 2011 - 2013 pour poursuivre la thèse.

Pour tenir compte du fait que nous serons amené à prendre en compte des variables retardées dans les modèles employés à la deuxième de la thèse, nous avons retenu une cohorte de 1525 entreprises pour la période 2011 2013 afin d'analyser aussi bien les seuils que les accruals. Après les différentes simulations et dans le souci d'avoir le maximum d'observations possibles, nous avons exclu de notre période d'étude les années 2010, 2014 et 2015. La période retenue va de 2011 à 2013. Ceci fait, notre cohorte finale se présente ainsi qu'il suit : 
Tableau 4 : Constitution de la cohorte finale

\begin{tabular}{|c|c|c|c|c|}
\hline & $\mathbf{2 0 1 1}$ & $\mathbf{2 0 1 2}$ & $\mathbf{2 0 1 3}$ & $\begin{array}{c}\text { Total des } \\
\text { Observations }\end{array}$ \\
\hline Échantillon de départ & 4193 & 6170 & 5708 & 16071 \\
\hline Données non disponibles sur les 03 années d'étude & 2668 & 4645 & 4183 & 11496 \\
\hline Cohorte finale & 1525 & 1525 & 1525 & 4575 \\
\hline
\end{tabular}

\section{$\underline{\text { Source : par nos soins }}$}

Nous n'avons retenu que les entreprises présentant des comptes sur les 03 exercices que nous avons retenus. Nous avons retiré les entreprises présentant des valeurs manquantes sur les postes que nous avions besoin d'étudier à savoir résultat avant impôt, chiffre d'affaires, ou total actif. Le tableau ci-dessus décrit la répartition par année des entreprises de notre échantillon. Il convient de noter que notre cohorte comporte finalement 1525 observations pour chaque année de l'étude. Par la suite, nous allons éliminer les observations extrêmes par rapport à la moyenne. Il est intéressant de présenter la répartition de notre échantillon par secteur d'activité selon la nomenclature de l'institut national de la statistique du Cameroun. Cette nomenclature distingue les entreprises du secteur primaire (codes 0001 et 0007), les entreprises du secteur secondaire (codes 0008 et 0030) et les entreprises du secteur tertiaire (codes 0031 et 0047).

Tableau 5 : Répartition des entreprises par secteur d'activité

\begin{tabular}{|l|r|r|r|l|r|}
\hline & $\mathbf{2 0 1 1}$ & $\mathbf{2 0 1 2}$ & $\mathbf{2 0 1 3}$ & $\begin{array}{l}\text { Total des } \\
\text { Observations }\end{array}$ & \multicolumn{2}{l|}{ Pourcentage } \\
\hline Échantillon final & 1525 & 1525 & 1525 & 4575 & $100 \%$ \\
\hline Secteur primaire & 33 & 33 & 33 & 99 & $2,16 \%$ \\
\hline Secteur secondaire & 178 & 178 & 178 & 534 & $11,67 \%$ \\
\hline Secteur tertiaire & 1314 & 1314 & 1314 & 3942 & $86,16 \%$ \\
\hline
\end{tabular}

Source : par nos soins

Selon le tableau ci-dessus 86, $16 \%$ des entreprises de notre échantillon sont constituées des entreprises du secteur du tertiaire contre $11,67 \%$ pour le secteur secondaire et $2,16 \%$ pour les entreprises du secteur primaire. Cette répartition est caractéristique de l'économie camerounaise qui est majoritairement dominée par les entreprises du tertiaire (commerce de gros et détails, activités de réparation, hôtellerie et restauration, transport et services associés, communication, postes et télécommunications, prestations de services aux entreprises entre autres).

Une fois la répartition par secteurs d'activités effectuée, il est également conseillé par les autorités scientifiques de décliner la répartition géographique des entreprises de notre échantillon. Faute d'obtenir dans les délais les informations plus précises sur la géographie des entreprises de notre échantillon, nous allons nous contenter de la répartition par régions.

Tableau 6 : Répartition des entreprises par régions

\begin{tabular}{|c|c|c|c|c|c|}
\hline & $\mathbf{2 0 1 1}$ & $\mathbf{2 0 1 2}$ & $\mathbf{2 0 1 3}$ & $\begin{array}{c}\text { Total des } \\
\text { Observations }\end{array}$ & Pourcentage \\
\hline $\begin{array}{c}\text { Échantillon } \\
\text { final }\end{array}$ & 1525 & 1525 & 1525 & 4575 & $100 \%$ \\
\hline Adamaoua & 17 & 17 & 17 & 51 & $1,11 \%$ \\
\hline Centre & 571 & 571 & 571 & 1713 & $37,44 \%$ \\
\hline Dont Yaoundé & 548 & 548 & 548 & 1644 & $35,93 \%$ \\
\hline Est & 16 & 16 & 16 & 48 & $1,05 \%$ \\
\hline Extrême nord & 21 & 21 & 21 & 63 & $1,38 \%$ \\
\hline Littoral & 643 & 643 & 643 & 1929 & $42,16 \%$ \\
\hline Dont Douala & 590 & 588 & 588 & 1766 & $38,60 \%$ \\
\hline Nord & 26 & 26 & 26 & 78 & $1,70 \%$ \\
\hline Nord ouest & 48 & 48 & 48 & 144 & $3,15 \%$ \\
\hline Ouest & 100 & 100 & 100 & 300 & $6,56 \%$ \\
\hline Sud & 14 & 14 & 14 & 42 & $0,92 \%$ \\
\hline Sud ouest & 69 & 69 & 69 & 207 & $4,52 \%$ \\
\hline
\end{tabular}

Source : par nos soins

Selon le tableau ci-dessus nous constatons que environ 37, $44 \%$ des entreprises de notre échantillon sont implantées dans le centre dont environ $35,93 \%$ pour la seule ville de Yaoundé. Concernant la région du littoral, elle concentre près de $42,16 \%$ des entreprises dont $38,6 \%$ pour la seule ville de Douala. Ces chiffres militent pour la représentativité de notre échantillon car en plus d'avoir les entreprises de toutes les régions du Cameroun, elle 
est presque conforme aux statistiques du recensement général des entreprises réalisé par l'INS en 2016 et selon lequel Douala et Yaoundé cumulent plus trois quarts des entreprises recensées au Cameroun.

Après les détails sur la répartition géographique des entreprises de notre échantillon, il s'avère nécessaire de présenter également la répartition de nos entreprises en fonction de leur taille. La notion de taille est une notion polysémique dans la littérature. Certains auteurs retiennent comme critère de regroupement la taille. D'autres préfèrent le chiffre d'affaires ou encore l'effectif des employés. Pour le cas de cette thèse, nous avons choisi comme critère de regroupement le chiffre d'affaires car celui est une variable fondamentale dans notre thèse. En effet la manipulation des comptes étant principalement tournée vers la manipulation du résultat induit forcement une place de choix pour le chiffres d'affaires car celui-ci intervient dans le calcul du résultat. Le tableau ci-après synthétise le regroupement des entreprises de notre échantillon selon la taille. Les différentes classes de chiffre d'affaires sont inspirées de la classification établie par l'INS du Cameroun dans la centrale des bilans de 2016.

Tableau 7 : Répartition des entreprises par taille

\begin{tabular}{|c|c|c|c|c|c|}
\hline $\begin{array}{l}\text { Années } \\
\text { Chiffre d'affaires } \\
\end{array}$ & 2011 & 2012 & 2013 & $\begin{array}{c}\text { Total des } \\
\text { Observations }\end{array}$ & Pourcentage \\
\hline $\begin{array}{r}\text { Échantillon final } \\
\end{array}$ & 1525 & 1525 & 1525 & 4575 & $100 \%$ \\
\hline Moins de $\quad 15000000$ FCFA & 921 & 921 & 921 & 2763 & $60,39 \%$ \\
\hline De 15000000 à $\quad 50000000$ FCFA & 287 & 287 & 287 & 861 & $18,82 \%$ \\
\hline De 50000000 à $\quad 100000000$ FCFA & 136 & 136 & 136 & 408 & $8,92 \%$ \\
\hline $\begin{array}{r}\text { De } 100000000 \text { à } \\
250000000 \text { FCFA } \\
\end{array}$ & 78 & 78 & 78 & 234 & $5,11 \%$ \\
\hline $\begin{array}{c}\text { De } 250000000 \text { à } \\
1000000000 \text { FCFA }\end{array}$ & 55 & 55 & 55 & 165 & $3,61 \%$ \\
\hline $\begin{array}{l}\text { De } 1000000000 \text { à } \\
3000000000 \text { FCFA }\end{array}$ & 26 & 26 & 26 & 78 & $1,70 \%$ \\
\hline $\begin{array}{c}3000000000 \text { FCFA } \\
\text { et plus }\end{array}$ & 22 & 22 & 22 & 66 & $1,44 \%$ \\
\hline
\end{tabular}

\section{Source : par nos soins}

Après une description aussi rigoureuse que possible des caractéristiques de notre échantillon, nous présentons ci-dessus les méthodes statistiques utilisées pour effectuer nos analyses statistiques.

\subsection{Les méthodes statistiques mobilisées}

Pour tester nos hypothèses, nous utilisons 02 méthodes statistiques à savoir les histogrammes de normalité et les tests statistiques (Bursgstahler et Dichev, 1997). Nous utilisons les histogrammes de normalité pour observer les discontinuités dans la distribution des résultats afin de déduire les entreprises suspectes. L'identification des entreprises suspectes est réalisée en adaptant la démarche de Roychowdhry (2006). Ainsi, dans notre repère nous représentons en abscisse le résultat avant impôt divisé par le total des actifs alors que l'axe des ordonnées désigne le nombre des entreprises.

Les tests statistiques utilisés dans le cadre de cette thèse proviennent de Bursgstahler et Dichev (1997). Il est question de comparer les effectifs observés avec les effectifs théoriques issues de la loi normale, un écart entre les deux effectifs traduisant l'existence de la manipulation même si la démarche est silencieuse sur qui manipule et combien il manipule.

\section{La présentation et l'interprétation des résultats}

Après la sélection des données et l'échantillonnage, nous procédons aux analyses et présentons les résultats de notre étude. Ces résultats sont ensuite interprétés afin de ressortir des conclusions.

\subsection{La préparation des données et l'identification des entreprises suspectes}

Pour construire les histogrammes des distributions des résultats nets divisés par le total des actifs, il faut étudier de manière approfondie les caractéristiques de cette variable à travers notre échantillon de 1525 entreprise et sur la période d'étude (2011 à 2013). L'Analyse statistique permet de définir une échelle adéquate pour la construction des histogrammes empiriques contenant le maximum d'observations dans la présentation de la densité de la distribution, à savoir : l'étendue de la région symétrique autour du seuil zéro, la largeur ou l'amplitude des bâtons des histogrammes.

Nous dressons les statistiques descriptives relatives à la variable « $R A I / A T »$. Le tableau suivant montre un nombre total de 4575 observations. Le nombre des observations disponibles par année est de 1525 . La moyenne du ratio «RAI/AT» est négative sur toute la période d'étude. Pratiquement, la variation est positive entre 2011 et 2012, négative entre 2012 et 2013. 
Ce qui laisse penser que la propension des entreprises à publier des pertes varie d'une année à l'autre. Le mode est égal à zéro sur toute la période d'étude. Ce qui traduit le fait que l'écrasante majorité des entreprises publie les résultats pratiquement nuls. La médiane est positive et varie très peu entre les années d'étude. Nous remarquons que la moyenne et la médiane du ratio «RAI/AT» sont de signes opposés sur la période d'étude.

Le quartile moyen de premier ordre $(\mathrm{Q} 1)$ du ratio «RAI/AT» est négatif et va $-0,0781$ à $-0,1069$ alors que le quartile moyen de $3^{\mathrm{e}}$ ordre est positif et décroissant de 2011 à 2013. Les coefficients d'asymétrie et d'aplatissement sont non nuls sur toute la période d'étude.

Ce qui traduit que notre série statistique ne suit pas une loi normale. Il y aurait donc des entreprises qui manipulent leurs comptes dans notre série statistique pour atteindre ou franchir des seuils particuliers selon les prédictions de Burgstahler et Dichev (1997).

Tableau 8: Les statistiques descriptives de la variable «RAI/AT» sur la période de 2011 à 2013.

\begin{tabular}{|l|l|l|l|}
\hline STATISTIQUES ANNEES & \multicolumn{1}{|c|}{$\mathbf{2 0 1 1}$} & $\mathbf{2 0 1 2}$ & \multicolumn{1}{|c|}{$\mathbf{2 0 1 3}$} \\
\hline $\mathrm{N}$ & 1525 & 1525 & 1525 \\
\hline Moyenne &,- 0822 &,- 0785 &,- 1191 \\
\hline Médiane &, 0046 &, 0078 &, 0043 \\
\hline Mode & 0,00 & 0,00 & 0,00 \\
\hline Ecart-type &, 42463 &, 40931 &, 49323 \\
\hline Variance &, 180 &, 168 &, 243 \\
\hline Asymétrie & $-4,845$ & $-5,040$ & $-4,572$ \\
\hline Erreur std. d'asymétrie &, 063 &, 063 &, 063 \\
\hline Aplatissement & 33,560 & 37,416 & 27,975 \\
\hline Erreur std. d'aplatissement &, 125 &, 125 &, 125 \\
\hline Intervalle & 5,29 & 5,65 & 5,70 \\
\hline Minimum & $-4,30$ & $-4,74$ & $-4,74$ \\
\hline Maximum &, 99 &, 92 &, 97 \\
\hline Quartiles &,- 0987 &,- 0781 &,- 1069 \\
\hline &, 0046 &, 0078 &, 0043 \\
\hline
\end{tabular}

Ce tableau représente en ligne les paramètres statistiques et en colonne les années, la ligne ' $N$ ' représente le nombre des observations disponibles au titre de chaque année, les lignes suivantes représentent respectivement les valeurs annuelles du mode, de la moyenne, de l'écart type, du coefficient d'asymétrie, et d'autres paramètres de dispersion comme le premier quartile, la médiane et le $3^{\mathrm{e}}$ quartile.

$\underline{\text { Par nos soins }}$

Suite à cette brève description, nous constatons que nos histogrammes empiriques de la variable «RAI/AT» se trouvent dans la région symétrique $[-4,3 ; 0,99]$ en $2011 ;[-4,74 ; 0,92]$ en 2012 et $[-4,74 ; 0,97]$ en 2013. Les figures 9 à 11 représentent l'histogramme historique de la variable «RAI/AT» entre 2011 et 2013 et portant sur 1 525 entreprises ayant les données disponibles sur la période d'étude. L'axe des abscisses, une demi-droite symétrique par rapport à zéro, allant de - 4,74 à 1, est divisé en des intervalles égaux de 0,1 d'amplitude, représentant la largeur des bâtons qui composent les histogrammes. L'axe des ordonnées représente la fréquence absolue des observations dans chaque intervalle.

La figure ci-dessous montre une discontinuité au niveau de la distribution du ratio «RAI/AT» à un seuil égal à 0.00 autour de seuil zéro. Les fréquences de $R A I / A T$ dans les intervalles à gauche de zéro sont anormalement faibles alors que les fréquences avoisinant le seuil zéro sont anormalement élevées.

La poursuite de la même méthodologie basée sur les distributions du ratio «RAI/AT», nous permet d'identifier les entreprises qui gèrent leurs résultats - qualifiées d'entreprises suspectes. Dans une première étape, nous construisons les histogrammes empiriques annuels relatifs au ratio «RAI/AT». Nous obtenons trois distributions présentées dans les figures 9 à 11 .

Les résultats obtenus de la distribution des résultats avant impôt des entreprises camerounaises sont reportés dans les figures 9 à 11. Ces résultats avant impôt ont été normalisés par l'actif net pour la période allant de 2011 à 2013. La distribution des résultats est réalisée avec une amplitude de $10 \%$ afin d'avoir une distribution symétrique. 
Figure 3 : L'histogramme empirique de la distribution des résultats avant impôt normalisés par le total des actifs pour l'exercice 2011

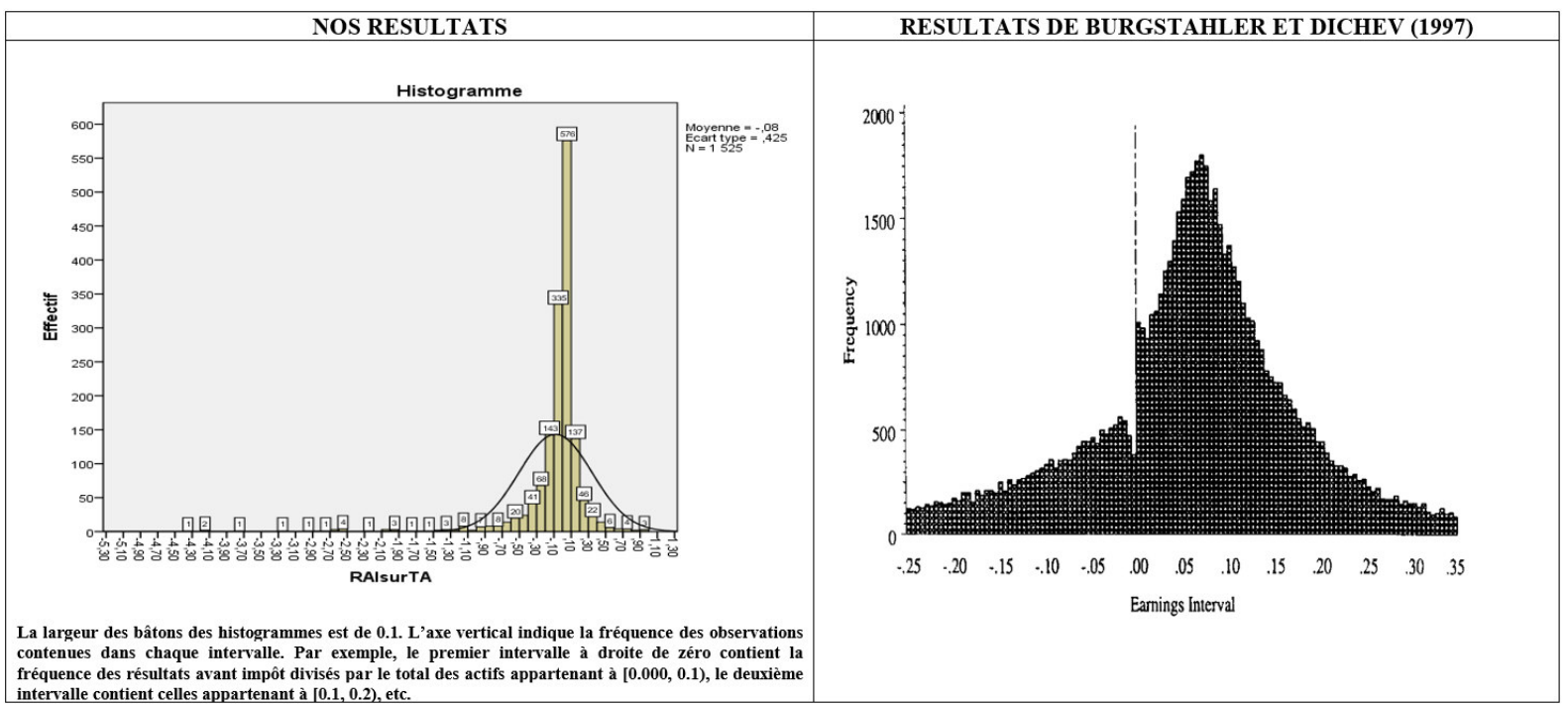

Figure 4 : L'histogramme empirique de la distribution des résultats avant impôt normalisés par le total des actifs pour l'exercice 2012

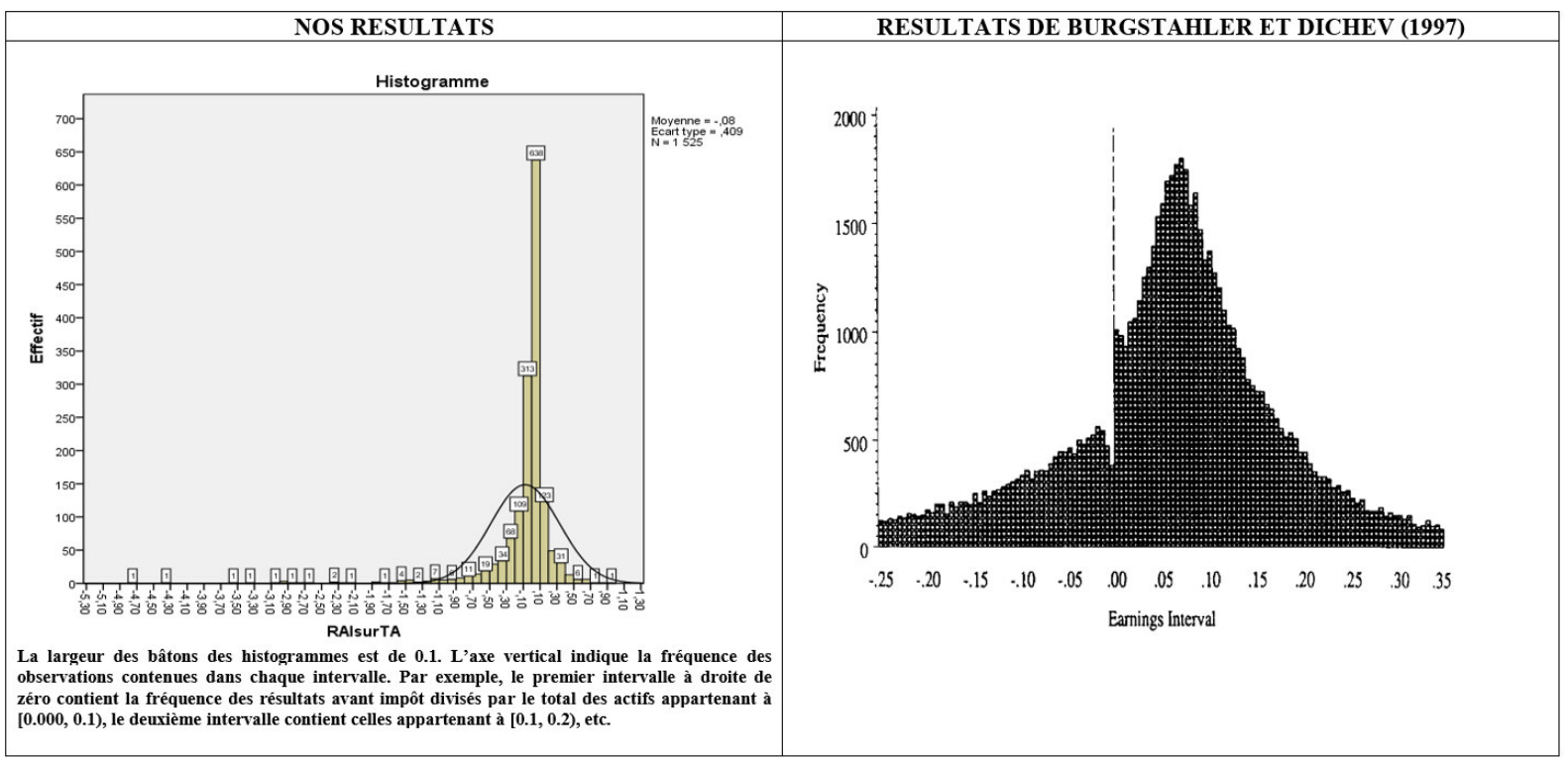


Figure 5 : L'histogramme empirique de la distribution des résultats avant impôt normalisés par le total des actifs pour l'exercice 2013.

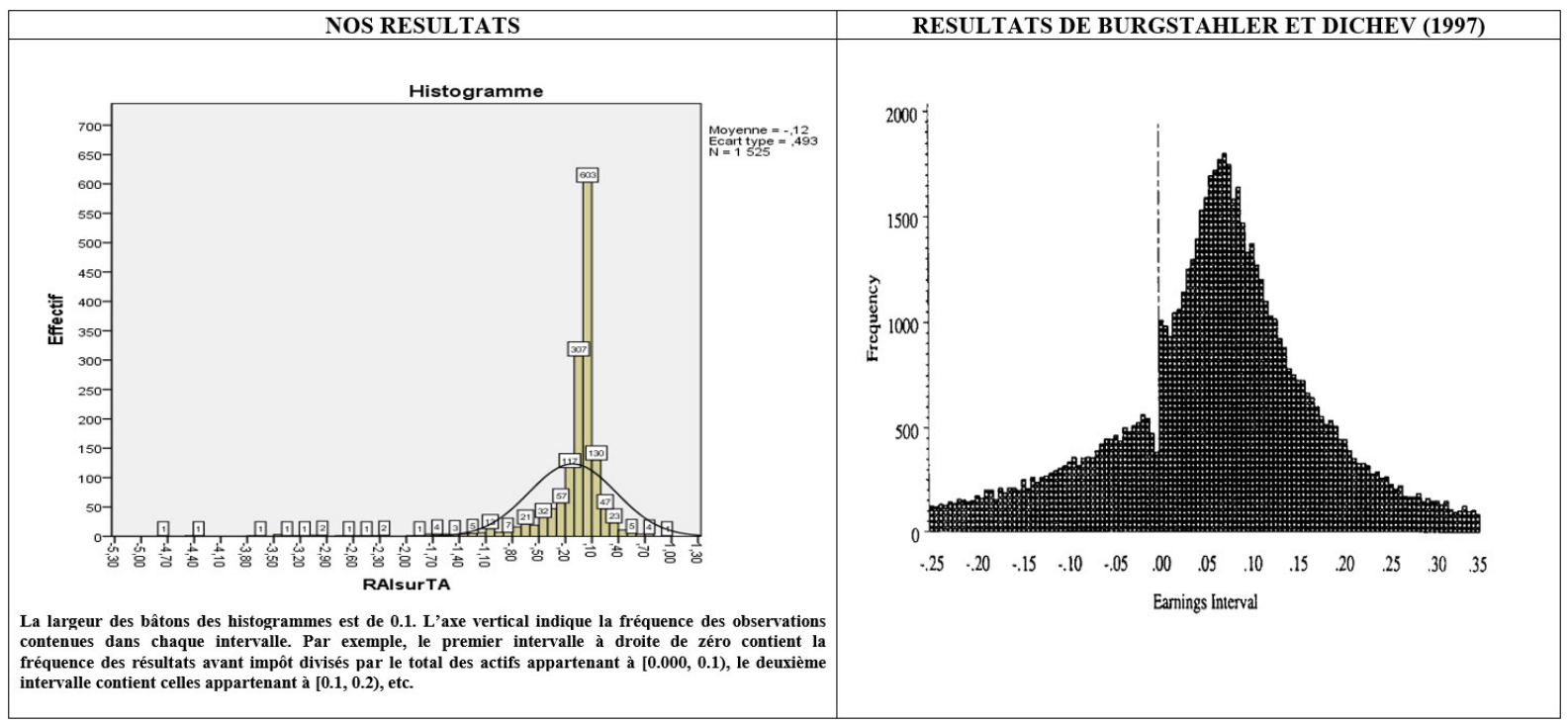

Les figures ci-dessus présentent la distribution statistique des résultats des 1525 entreprises de notre échantillon sur une période de trois ans. Ces résultats ont été mis en perspective avec les résultats de Burgstahler et Dichev (1997). Le principal enseignement à mettre en évidence est qu'une discontinuité est bien constatée au niveau du résultat nul. Ce qui traduit bien la présence d'un effet seuil chez les entreprises implantées au Cameroun. Les intervalles $[-0,1 ; 0]$ et $[0 ; 0,1]$ concentrent plus d'entreprises que les autres intervalles. Tableau 9 : Nombre des entreprises suspectes et non suspectes et leurs caractéristiques

\begin{tabular}{|c|c|c|c|c|c|c|c|c|c|c|c|}
\hline & \multicolumn{3}{|c|}{ toutes les entreprises } & \multicolumn{4}{|c|}{ Entreprises suspectes } & \multicolumn{4}{|c|}{ Entreprises non suspectes } \\
\hline Années & $\mathrm{N}$ & $\begin{array}{c}\text { Moyen } \\
\text { ne }\end{array}$ & $\begin{array}{l}\text { Ecart- } \\
\text { type }\end{array}$ & $\mathrm{N}$ & $\%$ & $\begin{array}{c}\text { Moyen } \\
\text { ne }\end{array}$ & $\begin{array}{l}\text { Ecart- } \\
\text { type }\end{array}$ & $\mathrm{N}$ & $\%$ & $\begin{array}{c}\text { Moyen } \\
\text { ne }\end{array}$ & $\begin{array}{l}\text { Ecart- } \\
\text { type }\end{array}$ \\
\hline 2011 & 1525 &,- 0822 & ,42463 & 1060 & $\begin{array}{c}69,51 \\
\%\end{array}$ &, 0251 & ,06479 & 465 & $\begin{array}{c}30,49 \\
\%\end{array}$ &,- 3269 & ,70450 \\
\hline 2012 & 1525 &,- 0785 & ,40931 & 962 & $\begin{array}{c}63,08 \\
\%\end{array}$ & ,0082 & ,04439 & 563 & $\begin{array}{c}36,92 \\
\%\end{array}$ &,- 2264 & ,64510 \\
\hline 2013 & 1525 &,- 1191 & ,49323 & 1049 & $\begin{array}{c}68,79 \\
\% \\
\end{array}$ & ,0246 & ,06333 & 476 & $\begin{array}{c}31,21 \\
\%\end{array}$ &,- 4357 & ,79097 \\
\hline TOTAUX & 4575 & & & 3071 & & & & 1504 & & & \\
\hline $\begin{array}{c}\text { FREQUEN } \\
\text { CE }\end{array}$ & \multicolumn{3}{|l|}{$\begin{array}{c}100,00 \\
\%\end{array}$} & \multicolumn{4}{|l|}{$\begin{array}{c}67,13 \\
\%\end{array}$} & \multicolumn{4}{|l|}{$\begin{array}{c}32,87 \\
\%\end{array}$} \\
\hline
\end{tabular}

Source : Par nos soins

Le tableau ci-dessus récapitule le nombre et les caractéristiques respectivement des entreprises suspectes et des autres entreprises (non suspectes). Ce tableau nous permet de constater que les entreprises suspectes représentent $69,51 \%$ des entreprises de notre échantillon contre 30,41\% pour les entreprises non suspectes en 2011. La proportion d'entreprises suspectes baisse à 63,08\% et celle des entreprises non suspectes augmente pour atteindre 39,02\% en 2012. La tendance est renversée en 2013 où la fréquence des entreprises suspectes passe à $68,79 \%$ et celle des entreprises non suspectes à 31,21\%. Ce qui nous permet de valider le point de vue selon lequel la manipulation est prépondérante dans le contexte camerounais. Aussi, le transfert des entreprises suspectes vers les entreprises non suspectes est constaté entre 2011 et 2012 alors qu'entre 2012 et 2013, il y a transfert des entreprises non suspectes les entreprises suspectes. Ceci semble valider le fait que la manipulation intervenu en $\mathrm{N}$ produit des effets inverses en N+1 (Stolowy et Breton, 2003).

En comparant les moyennes des résultats avant impôt des différents groupes, nous constatons que les moyennes sont toutes négatives pour toutes les entreprises sur toute la période d'étude. Par contre pour les entreprises suspectes la moyenne est positive sur la même période. Les entreprises non suspectes quant à elles ont toutes les moyennes négatives. On peut donc penser que les entreprises suspectes manipulent leurs résultats en général à la hausse que ce soit pour publier des faibles pertes ou des faibles profits confortement à Burgstahler et Dichev (1997).

D'après les histogrammes et le tableau ci-dessus, nous pouvons faire un ensemble de conclusions. D'abord, 
nous observons une discontinuité dans la distribution du ratio «RAI/AT» autour du seuil zéro. Ensuite, les entreprises suspectes se situent dans l'intervalle $[-0,2 ; 0,2]$ en général. Enfin, le nombre total des observations suspectes est de 3071, représentant 67,13\% de l'échantillon de notre étude sur la période 2011-2013. Nous remarquons que la proportion des entreprises suspectes représente plus de la moitié des observations chaque année. Ceci confirme une forte prépondérance du phénomène de la manipulation dans un contexte où les entreprises cherchent à minimiser les transferts fiscaux. Notre hypothèse 1 est ainsi partiellement validée. Autrement dit, selon notre étude, l'approche par le seuil du résultat nul permet de détecter les entreprises suspectes et les entreprises non suspectes. Toutefois l'analyse sera poursuivie dans les sections suivantes pour détecter les entreprises manipulatrices pour compléter le test de notre hypothèse 1 .

Il est intéressant de peaufiner l'analyse en effectuant des tests de différences entre les entreprises suspectes et les entreprises non suspectes. Deux catégories de tests statistiques s'offrent à nous à cet effet: les tests paramétriques et les tests non paramétriques. Nous optons pour les tests non paramétriques car nos données ne remplissent pas les conditions exigées : normalité de la distribution, variances égales, échantillons de taille identique, homogénéité de la population. Les tests sont présentés dans le tableau ci-après :

Tableau 10 : tests de différences entre les entreprises suspectes et les entreprises non suspectes

\begin{tabular}{|c|c|c|c|c|c|c|c|}
\hline & \multicolumn{2}{|c|}{ Entreprises suspectes } & \multicolumn{2}{|c|}{ Entreprises non suspectes } & \multicolumn{3}{|c|}{ Test de différence } \\
\hline Années & $\mathrm{N}$ & Moyenne & $\mathrm{N}$ & Moyenne & test choisi & Sig & Décision \\
\hline 2011 & 1060 & 0,0251 & 465 & $-0,3269$ & $\begin{array}{c}\text { U de Mann } \\
\text { Whitney }\end{array}$ & 0,0000 & $\begin{array}{c}\text { échantillons } \\
\text { indépendants à un seuil } \\
\text { inférieur à 5\% }\end{array}$ \\
\hline 2012 & 962 & 0,0082 & 563 & $-0,2264$ & $\begin{array}{c}\text { U de Mann } \\
\text { Whitney }\end{array}$ & 0,0000 & $\begin{array}{c}\text { échantillons } \\
\text { indépendants à un seuil } \\
\text { inférieur à } 5 \%\end{array}$ \\
\hline 2013 & 1049 & 0,0246 & 476 & $-0,4357$ & $\begin{array}{c}\text { U de Mann } \\
\text { Whitney }\end{array}$ & 0,000 & $\begin{array}{c}\text { échantillons } \\
\text { indépendants au seuil de } \\
5 \%\end{array}$ \\
\hline TOTAUX & 3071 & & 1504 & & & \\
\hline
\end{tabular}

Source : par nos soins

Selon le tableau ci-haut, nous pouvons bien conclure que le comportement des entreprises suspectes est différent de celui des entreprises non suspectes en matière de publication des résultats sur la période d'étude. Pour les années 2011, 2012 et 2013 le comportement des entreprises suspectes est bien différent de celui des entreprises non suspectes à un seuil inférieur à $5 \%$.

Ayant présenté les résultats concernant l'identification des entreprises non suspectes et des entreprises suspectes, sur la base de l'étude de la distribution des résultats, l'analyse est affinée en mettant en évidence les observations attendues avant de déterminer les firmes qui ont manipulé leurs résultats comptables, en analysant l'évolution postérieure de leurs résultats. Par la suite, la seconde hypothèse, selon laquelle la proportion d'entreprises qui publie les faibles profits est plus élevée que celle des entreprises publiant les faibles pertes est traitée.

Tableau 11 : Comparaison de la proportion des entreprises publiant les faibles pertes avec la proportion des entreprises publiant les faibles profits

\begin{tabular}{|c|c|c|c|c|}
\hline & \multicolumn{2}{|c|}{$\begin{array}{c}\text { Entreprises suspectes à gauche du seuil } \\
\text { zéro }\end{array}$} & \multirow{2}{*}{ Entreprises suspectes à droite du seuil zéro } \\
\hline Années & $\mathrm{N}$ & Pourcentage & $\mathrm{N}$ & Pourcentage \\
\hline 2011 & 336 & $32,03 \%$ & 713 & $67,97 \%$ \\
\hline 2012 & 317 & $32,95 \%$ & 645 & $67,05 \%$ \\
\hline 2013 & 307 & $29,52 \%$ & 733 & $70,48 \%$ \\
\hline
\end{tabular}

Source : par nos soins

D'après le tableau ci-dessus, la proportion des entreprises suspectes se trouvant à droite du seuil zéro est supérieure à la proportion des entreprises suspectes se trouvant à gauche du seuil sur toute la période d'étude. Notre hypothèse 2 est donc validée. En d'autres termes, par rapport à la population totale, la proportion d'entreprises suspectes publiant les faibles profits est supérieure à la proportion d'entreprises suspectes publiant les faibles pertes. Ce résultat est conforme à celui de Grima (2017) qui trouve que dans le contexte français, la proportion d'entreprises suspectes se trouvant à droite du seuil est supérieure à la proportion d'entreprises se trouvant à gauche du seuil.

Après l'analyse de la distribution des résultats avant impôt, les données seront testées telles qu'elles auraient dû l'être, avant de mettre en relief les sociétés ayant manipulé leurs résultats, grâce à l'analyse de leurs résultats futurs. 
Tableau 12 : Extrait de la distribution des entreprises en fonction des résultats

\begin{tabular}{|l|r|r|r|}
\hline & 2011 & 2012 & 2013 \\
\hline$<-\mathbf{0 , 4}$ & 124 & 132 & 169 \\
\hline$[-0,4 ;-0,3[$ & 41 & 34 & 47 \\
\hline$[-0,3 ;-0,2[$ & 68 & 68 & 57 \\
\hline$[-0,2 ;-0,1[$ & 143 & 109 & 117 \\
\hline$[-0,1 ; 0[$ & 335 & 313 & 307 \\
\hline$[0 ; 0,1[$ & 576 & 638 & 603 \\
\hline$[0,1 ; 0,2[$ & 137 & 123 & 130 \\
\hline$[0,2 ; 0,3[$ & 46 & 49 & 47 \\
\hline$[0,3 ; 0,4[$ & 22 & 31 & 23 \\
\hline$>=\mathbf{0}, \mathbf{4}$ & 33 & 28 & 25 \\
\hline TOTAL & 1525 & 1525 & 1525 \\
\hline
\end{tabular}

Source : Par nos soins

La distribution empirique, présente une anomalie au voisinage de zéro. En effet, considérons l'intervalle [$0,1 ; 0]$ qui présente un nombre d'entreprises de l'ordre de 335 en 2011 par exemple. Ce nombre signifie que 335 entreprises ont eu un résultat légèrement déficitaire en 2011. Logiquement, la distribution attendue des données comptables devrait suivre une distribution répartissant les valeurs de la même manière sur l'ensemble de l'étude. Or, pour l'intervalle symétrique $[0 ; 0,1]$, en 2011 , le nombre d'entreprises est de 576 , soit un effectif supérieur à celui des entreprises légèrement déficitaires. Dans le but de valider l'hypothèse selon laquelle les entreprises comprises dans cet intervalle bénéficiaire pourraient être suspectées d'avoir manipulé leurs résultats, il faut calculer le nombre d'observations théoriques de cet intervalle

\subsection{Distribution de résultats attendue au seuil de zéro}

Pour calculer les effectifs théoriques, nous utilisons la moyenne arithmétique (Burgstahler et Dichev, 1997b) : Il est question de prendre les effectifs des deux intervalles adjacents à celui étudié et de calculer la moyenne. Ceci donne :

$\mathrm{N}[-0,1 ; 0]$ attendu $=(\mathrm{N}[-\mathbf{0}, 2 ;-0,1]$ observé $+\mathrm{N}[0 ; 0,1]$ observé $) / 2$.

Cette formule peut être testée sur les différents intervalles de notre étude. Pour l'intervalle $[-0,1 ; 0]$ la valeur attendue de :

$\mathrm{N}[-1 ; 0]$ attendu $=(143+576) / 2=359,5$ que nous arrondissons à 360 puisqu'il n'est pas possible d'avoir 0,5 entreprise.

La valeur attendue ici est supérieure à la valeur observée, qui est de 335. Nous avons calculé la même moyenne pour les autres intervalles de l'étude dont il ressort une anomalie de distribution au voisinage de zéro, comme le montre le tableau suivant. Le tableau ne retient que les intervalles où une suspicion de manipulation a été établie à la lecture des histogrammes de distribution des résultats, soit l'intervalle [-0,1;0,2[ en 2011 et 2013 ; l'intervalle $[-0,1 ; 0,1[$ en 2012 .

Tableau 13 : Distribution théorique et distribution constatée des effectifs des entreprises en fonction des résultats de 2011 à 2013 dans l'intervalle de suspicion (de $-0,1$ à 0,2$)$

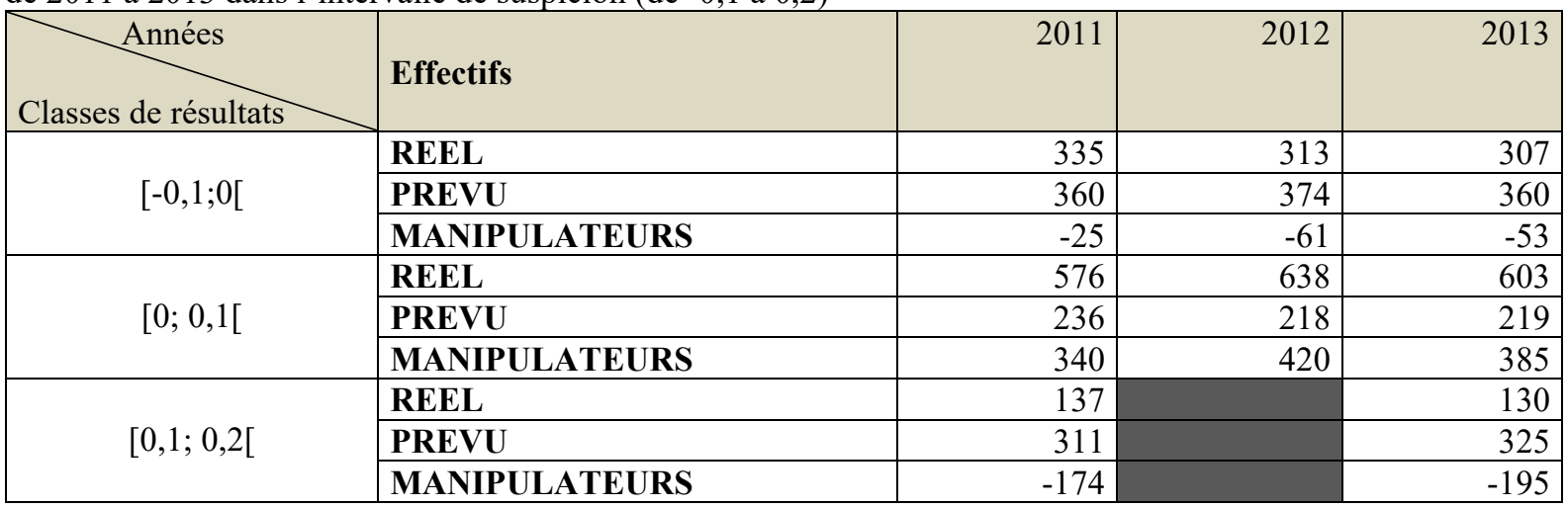

\section{Source: Par nos soins}

La différence la plus flagrante mise en évidence par le tableau correspond à la distribution du résultat avant impôt dans l'intervalle [ $0 ; 1]$. En effet, en 2011 par exemple nous avons 576 entreprises alors que les observations attendues étaient de :

$N[0 ; 1]$ attendu $=(335+137) / 2=236$

Il en découle que peu d'entreprises réalisent un faible déficit. Ce qui provoque l'interrogation sur les raisons de ces distributions anormales afin de déterminer les entreprises qui pratiquent la manipulation des chiffres 
comptables pour obtenir des conséquences précises.

\subsubsection{L'identification des entreprises manipulatrices}

Dans notre échantillon, nous conservons les entreprises qui présentent une distribution anormale de résultats et qui sont soupçonnées ici de manipulation. Dans un premier temps nous détaillons les tests utilisés pour savoir si notre échantillon contient des données manipulées. Sachant que la distribution des résultats manipulés est anormale autour de zéro, les firmes qui se trouvent dans cette zone, peuvent être soupçonnées de manipulation.

Après le trie des données, notre échantillon pour l'intervalle $[0 ; 1]$ est constitué de 576 entreprises suspectes en 2011 ; 638 entreprises suspectes en 2012 et 603 entreprises suspectes en 2013. Ces observations comprennent aussi bien les entreprises ayant un résultat proche du seuil de zéro du fait du hasard que les entreprises qui ont manipulé leur résultat pour atteindre ce seuil. La manipulation des chiffres comptables étant caractérisée par un report d'un résultat sur les exercices ultérieurs, et notre hypothèse étant de valider que des entreprises peuvent être suspectées de manipuler, nous allons déterminer l'évolution des résultats avant impôt pour les entreprises dans l'intervalle $[0 ; 1]$. Celles dont le résultat diminue ou reste stable pourront être considérées comme suspectes de manipuler à l'instant $\mathrm{T}$ leurs comptes. De même, nous affinerons notre analyse en analysant l'évolution du résultat sur l'année $\mathrm{N}+2$. En effet, une baisse du résultat $\mathrm{N}+1$ par rapport à $\mathrm{N}$, suivie d'une augmentation en $\mathrm{N}+2$, tendrait à confirmer que l'entreprise pourrait avoir eu recours à la gestion des données comptables en N (Grima, 2017).

4.2.2. L'évolution du résultat avant impôt pour les entreprises situées dans l'intervalle $[0 ; 1]$

L'évolution postérieure des résultats avant impôt des 576 entreprises de notre échantillon situées dans l'intervalle $[0 ; 1]$ nous permet de définir les firmes manipulatrices. La règle établie pour répartir notre échantillon peut être définie de la manière suivante. Lorsque le résultat en $\mathrm{N}+1$ est inférieur au résultat en $\mathrm{N}$ et que le résultat en $\mathrm{N}+2$ est supérieur au résultat en $\mathrm{N}+1$, il y a une forte probabilité de manipulation.

Pour appliquer cette règle nous devons, pour être rigoureux, suivre le comportement des entreprises suspectes identifiées en 2011 sur 03 années successives $(\mathrm{N}, \mathrm{N}+1$ et $\mathrm{N}+2)$. Ceci est primordial car nous devons analyser l'évolution des résultats sur trois exercices comptables. Notre cohorte comporte 576 entreprises à analyser pour chaque année. En éliminant celles ayant un résultat qui augmentait en $\mathrm{N}+1$ alors que nous attendions une baisse, il nous reste 349. Ensuite en soustrayant celles que leur résultat baissait en $\mathrm{N}+2$ alors que nous attendions une hausse, nous obtenons 200 entreprises pour lesquels la suspicion de manipulation est confirmée. Ces éléments sont récapitulés dans le tableau suivant.

Tableau 14 : Nombres d'entreprises ayant manipulé leurs chiffres comptables

\begin{tabular}{|l|l|l|l|}
\hline & $\begin{array}{l}\text { Cohorte des suspectes dans l'intervalle [0 ; } \\
\text { 0,1 pour la période 2011-2013 }\end{array}$ & RAI (2012inf2011) & RAI (2013sup2012) \\
\hline $\mathrm{N}$ & $\mathbf{5 7 6}$ & 349 & $\begin{array}{l}\text { 200 (nombre d'entreprises } \\
\text { manipulatrices) }\end{array}$ \\
\hline
\end{tabular}

\section{Source : Par nos soins}

Nous obtenons donc 200 entreprises manipulatrices sur la période 2011-2013, pour les entreprises publiant les faibles profits. Nous confrontons maintenant la tendance baissière du résultat en $\mathrm{N}+1$ et haussière en $\mathrm{N}+2$ avec les chiffres d'affaires sur la même période. Ce qui nous permet de conclure que l'approche par le seuil du résultat nul permet également d'identifier les entreprises manipulatrices. Par conséquent, notre hypothèse 1 est maintenant totalement validée avec la détection des entreprises manipulatrices.

Nos résultats sont conformes à ceux de Grima (2017) qui détecte également un nombre considérable d'entreprises manipulatrices parmi les entreprises suspectes.

\subsubsection{La comparaison entre les données observées et les données attendues}

En reprenant les éléments de départ, notre cohorte d'entreprises suspectes comportait 576 entreprises dans l'intervalle $[0 ; 1]$. Sur ces entreprises, nous avons obtenu un total de 200 entreprises manipulatrices. Le nombre de manipulations attendu du même intervalle est de 340 selon les tableaux précédents, ce qui fait un différentiel de 140 entreprises. Le nombre de manipulations observées (200) représente 58,82\% des manipulations attendues. Les éléments ci-dessus présentés sont détaillés dans le tableau ci-dessous.

Tableau 15: Comparaison des données observées avec les données attendues

\begin{tabular}{|l|l|}
\hline & Intervalle $0-0,1$ \\
\hline Observations attendues & 236 \\
\hline Observations réelles & 576 \\
\hline Suspicion de manipulation & 340 \\
\hline Détection de manipulation confirmée sur trois exercices & 200 \\
\hline Proportion de manipulation confirmée par rapport à la suspicion & $58,82 \%$ \\
\hline
\end{tabular}

Source : Par nos soins

\section{Conclusion}

Cette recherche a réalisé une étude empirique de la détection de la manipulation des chiffres comptables à travers 
le seuil du résultat nul. A l'aide des démarches de Burgstahler et Dichev (1997), Degeorge et al. (1999), Mard (2004), Grima (2017), nous avons effectué des tests empiriques sur un échantillon d'entreprises implantées au Cameroun. Ces tests ont été effectués pour tester nos deux premières de la thèse.

Nous avons opéré une tentative de mise en évidence la suspicion de manipulation des chiffres comptables perpétrée par les entreprises camerounaises afin d'orienter de façon discrétionnaire les résultats publiés. Sur la base d'une analyse croisée sur trois exercices consécutifs, nous avons confirmé la suspicion de manipulation et avons identifié les manipulateurs. Les tests effectués nous ont permis de valider nos hypothèses en prenant en considération un échantillon de 1525 entreprises-années. Nous avons émis l'hypothèse de discontinuité dans les observations des résultats comptables à l'approche du seuil de zéro. Ces prédictions sont susceptibles d'être validées puisque un pic de distribution est observé à un niveau très faiblement positif de résultat. En clair, le sort de nos hypothèses à la suite de l'épreuve des tests se présente de la manière suivante.

L'hypothèse 1 a été validée et nous permet d'affirmer que l'approche du seuil du résultat nul permet de détecter dans le contexte du Cameroun, les entreprises non suspectes, les entreprises suspectes et les entreprises manipulatrices.

L'hypothèse 2 a également été validée et débouche sur la conclusion selon laquelle la proportion d'entreprises suspectes situées à droite du seuil est supérieure à la proportion d'entreprises suspectes situées à gauche du seuil. Autrement dit la majorité des entreprises suspectes manipulent leurs résultats pour publier les faibles profits. Ce qui est conforme aux résultats de Burgstahler et Dichev (1997), Mard (2004), Vidal (2008), Grima (2017). Ceci semble surprenant eu égard aux motivations essentiellement orientées vers la minimisation des transferts fiscaux prêtées à la manipulation comptable en contexte camerounais. Ces motivations laissent penser que la majorité des entreprises suspectes devrait se trouver à gauche du seuil Zéro car les entreprises dans le contexte du Cameroun manipulent les comptes pour minimiser la base imposable (Ngantchou, 2008; Minko, 2016; Elle, 2018, Ngantchou et Elle, 2018).

Or il n'en est point le cas à ce niveau. En effet, nous soutenons que la minimisation de la base imposable ne veut pas forcement dire publier un résultat négatif. On peut minimiser la base imposable sans forcement publier les pertes. Aussi dans le contexte du Cameroun, la forte méfiance du fisc vis-à-vis des chiffres comptables a aboutit depuis plus d'une décennie à la neutralisation de l'impôt nul et de l'impôt négatif via l'algorithme du minimum de perception. Nous faisons référence à ce niveau à l'impôt sur le résultat des entreprises. Cette dynamique du minimum de perception nous amène à penser qu'en plus du seuil du résultat nul, il faut développer pour les recherches futures d'autres seuils mieux adaptés à l'environnement camerounais.

\section{Références bibliographiques}

BURGSTAHLER D. et DICHEV I. (1997), «Earnings management to avoid earnings decreases and losses », Journal of Acounting and Economics, vol 24, P. $99-126$.

BRETON G. et SCHATT A. (2003) «Les manipulations comptables : les dirigeants et les autres parties prenantes », Working Paper, Université du Quebec à Montreal, Canada.

DEGEORGE F. et al. (1999), "Earnings manipulations to exceed thresholds." Journal of Business Vol 72, P. 133.

ELLE N. (2018), La Manipulation des nombres comptables dans les entreprises au Cameroun : motivations et stratégies des acteurs, Thèse de Doctorat PhD, Université de Douala.

GRIMA C. (2017), Impacts des normes IFRS sur la manipulation comptable des sociétés françaises cotées, thèse de Doctorat PhD, CNAM, France.

HAYN C. (1995), "The information content of losses." Journal of Accounting and Economics 20 [2], P. 125-153.

MARD Y. (2004), "Les sociétés françaises cotées gèrent-elles leurs chiffres comptables afin d'éviter les pertes et les baisses de résultat? ", Comptabilité - Contrôle - Audit, Tome 10, Volume 2, P. 73-98.

MINKO G. (2016a), La détection des cas de manipulation comptables, Thèse de Doctorat PhD, Université de Douala.

NGANTCHOU A. (2008), «Recentrage du cadre comptable, durcissement de l'environnement fiscal et persistance de la gestion des données comptables : une étude du comportement des petites et moyennes entreprises camerounaises ", in Actes du $29^{e}$ congrès de l'AFC (Association Francophone de Comptabilité).

NGANTCHOU A. (2013), «L'influence du profil éthique du propriétaire dirigeant sur le contenu informatif des nombres comptables », Revue Internationnale PME, Vol. 26, $n^{\circ} 2, \quad$ P. 13-36.

NGANTCHOU A. et ELLE N. T. (2018) «La manipulation des chiffres comptables en contexte africain : la pertinence de l'hypothèse des 'coûts politiques"' », In Transitions numériques et comptabilités, $39^{e}$ Congres de l'AFC, Mai 2018, Nantes, France.

SAHA S. C. (2019), La détection de la manipulation des chiffres comptables : les perspectives de la réconciliation de l'approche par les seuils à l'approche par les accruals discrétionnaires, Thèse de Doctorat / PhD, Université de Douala.

SELLAMI M. et ADJAOUD F. (2010), « La gestion réelle des résultats : le cas des entreprises françaises », in 
Crises et nouvelles problématiques de la valeur, Nice, France.

VIDAL O. (2008), Gestion du résultat et seuils comptables : impact des choix méthodologiques et proposition d'un instrument de mesure des irrégularités, Thèse de Doctorat, HEC Paris, France.

VIDAL O. (2010), «Gestion du résultat pour éviter de publier une perte : les montants manipulés sont-ils marginaux ? », Comptabilité - Contrôle - Audit 2010/3 (Tome 16), p. 11-39. 